\title{
Planck intermediate results. XII: Diffuse Galactic components in the Gould Belt system ${ }^{\star}$
}

\author{
Planck Collaboration: P. A. R. Ade ${ }^{76}$, N. Aghanim ${ }^{51}$, M. I. R. Alves ${ }^{51}$, M. Arnaud ${ }^{65}$, M. Ashdown ${ }^{61,6}$, \\ F. Atrio-Barandela ${ }^{17}$, J. Aumont ${ }^{51}$, C. Baccigalupi ${ }^{75}$, A. Balbi ${ }^{31}$, A. J. Banday ${ }^{81,9}$, R. B. Barreiro ${ }^{58}$, J. G. Bartlett ${ }^{1,59}$, \\ E. Battaner ${ }^{83}$, L. Bedini ${ }^{8}$, K. Benabed ${ }^{52,80}$, A. Benoît ${ }^{49}$, J.-P. Bernard $^{9}$, M. Bersanellii ${ }^{29,43}$, A. Bonaldi ${ }^{60, \star \star}$, \\ J. R. Bond ${ }^{7}$, J. Borrill ${ }^{12,77}$, F. R. Bouchet ${ }^{52,80}$, F. Boulanger ${ }^{51}$, C. Burigana ${ }^{42,27}$, R. C. Butler ${ }^{42}$, P. Cabella ${ }^{32}$, \\ J.-F. Cardoso ${ }^{66,1,52}$, X. Chen ${ }^{48}$, L.-Y Chiang ${ }^{54}$, P. R. Christensen ${ }^{73,33}$, D. L. Clements ${ }^{47}$, S. Colombi ${ }^{52,80}$,
} L. P. L. Colombo 22,59 , A. Coulais ${ }^{64}$, F. Cuttaia ${ }^{42}$, R. D. Davies ${ }^{60}$, R. J. Davis ${ }^{60}$, P. de Bernardis ${ }^{28}$, G. de Gasperis ${ }^{31}$, G. de Zotti ${ }^{38,75}$, J. Delabrouille ${ }^{1}$, C. Dickinson ${ }^{60}$, J. M. Diego ${ }^{58}$, G. Dobler ${ }^{62}$, H. Dole ${ }^{51,50}$, S. Donzelli ${ }^{43}$, O. Doré ${ }^{59,10}$, M. Douspis ${ }^{51}$, X. Dupac ${ }^{35}$, T. A. Enßlin ${ }^{70}$, F. Finelli ${ }^{42,44}$, O. Forni ${ }^{81,9}$, M. Frailis ${ }^{40}$, E. Franceschi ${ }^{42}$, S. Galeotta ${ }^{40}$, K. Ganga ${ }^{1}$, R. T. Génova-Santos ${ }^{57}$, T. Ghosh ${ }^{51}$, M. Giard ${ }^{81,9}$, G. Giardino ${ }^{36}$, Y. Giraud-Héraud ${ }^{1}$,

J. González-Nuevo ${ }^{58,75}$, K. M. Górski ${ }^{59,85}$, A. Gregorio ${ }^{30,40}$, A. Gruppuso ${ }^{42}$, F. K. Hansen ${ }^{56}$, D. Harrison ${ }^{55,61}$, C. Hernández-Monteagudo ${ }^{11,70}$, S. R. Hildebrandt ${ }^{10}$, E. Hivon ${ }^{52,80}$, M. Hobson ${ }^{6}$, W. A. Holmes ${ }^{59}$, A. Hornstrup ${ }^{15}$, W. Hovest ${ }^{70}$, K. M. Huffenberger ${ }^{84}$, T. R. Jaffe ${ }^{81,9}$, A. H. Jaffe ${ }^{47}$, M. Juvela ${ }^{23}$, E. Keihänen ${ }^{23}$, R. Keskitalo ${ }^{20,12}$, T. S. Kisner ${ }^{69}$, J. Knoche ${ }^{70}$, M. Kunz ${ }^{16,51,3}$, H. Kurki-Suonio ${ }^{23,37}$, G. Lagache ${ }^{51}$, A. Lähteenmäki ${ }^{2,37}$, J.-M. Lamarre ${ }^{64}$,

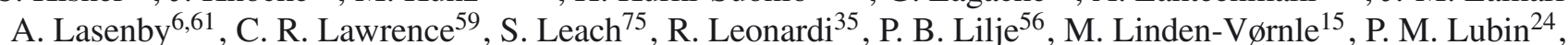
J. F. Macías-Pérez ${ }^{67}$, B. Maffei ${ }^{60}$, D. Maino ${ }^{29,43}$, N. Mandolesi ${ }^{42,5,27}$, M. Maris ${ }^{40}$, D. J. Marshall ${ }^{65}$, P. G. Martin ${ }^{7}$, E. Martínez-González ${ }^{58}$, S. Masi ${ }^{28}$, M. Massardi ${ }^{41}$, S. Matarrese ${ }^{26}$, P. Mazzotta ${ }^{31}$, A. Melchiorri2 ${ }^{28,45}$, A. Mennella ${ }^{29,43}$, S. Mitra ${ }^{46,59}$, M.-A. Miville-Deschênes ${ }^{51,7}$, A. Moneti ${ }^{52}$, L. Montier $^{81,9}$, G. Morgante ${ }^{42}$, D. Mortlock ${ }^{47}$, D. Munshi ${ }^{76}$, J. A. Murphy ${ }^{72}$, P. Naselsky ${ }^{73,33}$, F. Nati2 ${ }^{28}$, P. Natoli2 ${ }^{27,4,42}$, H. U. Nørgaard-Nielsen ${ }^{15}$, F. Noviello ${ }^{60}$, D. Novikov ${ }^{47}$,

I. Novikov ${ }^{73}$, S. Osborne ${ }^{79}$, C. A. Oxborrow ${ }^{15}$, F. Pajot ${ }^{51}$, R. Paladini ${ }^{48}$, D. Paoletti ${ }^{42,44}$, M. Peel ${ }^{60}$, L. Perotto ${ }^{67}$,

F. Perrotta ${ }^{75}$, F. Piacentini ${ }^{28}$, M. Piat ${ }^{1}$, E. Pierpaoli ${ }^{22}$, D. Pietrobon ${ }^{59}$, S. Plaszczynski ${ }^{63}$, E. Pointecouteau ${ }^{81,9}$, G. Polenta ${ }^{4,39}$, L. Popa ${ }^{53}$, T. Poutanen ${ }^{37,23,2}$, G. W. Pratt ${ }^{65}$, S. Prunet ${ }^{52,80}$, J.-L. Puget ${ }^{51}$, J. P. Rachen ${ }^{19,70}$,

W. T. Reach ${ }^{82}$, R. Rebolo ${ }^{57,13,34}$, M. Reinecke ${ }^{70}$, C. Renault ${ }^{67}$, S. Ricciardi ${ }^{42}$, I. Ristorcelli ${ }^{81,9}$, G. Rocha $^{59,10}$, C. Rosset $^{1}$, J. A. Rubiño-Martín ${ }^{57,34}$, B. Rusholme ${ }^{48}$, E. Salerno ${ }^{8}$, M. Sandri ${ }^{42}$, G. Savini ${ }^{74}$, D. Scott ${ }^{21}$, L. Spencer ${ }^{76}$, V. Stolyarov ${ }^{6,61,78}$, R. Sudiwala ${ }^{76}$, A.-S. Suur-Uski ${ }^{23,37}$, J.-F. Sygnet ${ }^{52}$, J. A. Tauber $^{36}$, L. Terenzi ${ }^{42}$, C. T. Tibbs ${ }^{48}$,

L. Toffolatti ${ }^{18,58}$, M. Tomasi ${ }^{43}$, M. Tristram ${ }^{63}$, L. Valenziano $^{42}$, B. Van Tent ${ }^{68}$, J. Varis ${ }^{71}$, P. Vielva ${ }^{58}$, F. Villa ${ }^{42}$, N. Vittorio ${ }^{31}$, L. A. Wade ${ }^{59}$, B. D. Wandelt ${ }^{22,80,25}$, N. Ysard ${ }^{23}$, D. Yvon ${ }^{14}$, A. Zacchei $^{40}$, and A. Zonca ${ }^{24}$

(Affiliations can be found after the references)

Received 24 January 2013 / Accepted 31 May 2013

\section{ABSTRACT}

\begin{abstract}
We perform an analysis of the diffuse low-frequency Galactic components in the southern part of the Gould Belt system $\left(130^{\circ} \leq l \leq 230^{\circ}\right.$ and $-50^{\circ} \leq b \leq-10^{\circ}$ ). Strong ultra-violet flux coming from the Gould Belt super-association is responsible for bright diffuse foregrounds that we observe from our position inside the system and that can help us improve our knowledge of the Galactic emission. Free-free emission and anomalous microwave emission (AME) are the dominant components at low frequencies $(v<40 \mathrm{GHz})$, while synchrotron emission is very smooth and faint. We separated diffuse free-free emission and AME from synchrotron emission and thermal dust emission by using Planck data, complemented by ancillary data, using the correlated component analysis (CCA) component-separation method and we compared our results with the results of cross-correlation of foreground templates with the frequency maps. We estimated the electron temperature $T_{\mathrm{e}}$ from $\mathrm{H} \alpha$ and free-free emission using two methods (temperature-temperature plot and cross-correlation) and obtained $T_{\mathrm{e}}$ ranging from 3100 to $5200 \mathrm{~K}$ for an effective fraction of absorbing dust along the line of sight of $30 \%\left(f_{\mathrm{d}}=0.3\right)$. We estimated the frequency spectrum of the diffuse AME and recovered a peak frequency (in flux density units) of $25.5 \pm 1.5 \mathrm{GHz}$. We verified the reliability of this result with realistic simulations that include biases in the spectral model for the AME and in the free-free template. By combining physical models for vibrational and rotational dust emission and adding the constraints from the thermal dust spectrum from Planck and IRAS, we are able to present a good description of the AME frequency spectrum for plausible values of the local density and radiation field.
\end{abstract}

Key words. Galaxy: general - radio continuum: ISM - radiation mechanisms: general

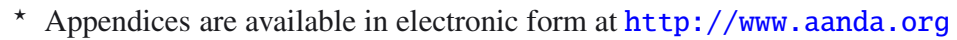

$\star \star$ Corresponding author: A. Bonaldi (e-mail: anna.bonaldi@manchester.ac.uk)
} 


\section{Introduction}

The wide frequency coverage of the Planck $^{1}$ data provides a unique opportunity of studying the main four Galactic foregrounds, namely free-free emission, synchrotron emission, anomalous microwave emission (AME), and thermal (vibrational) dust emission. The different frequency spectra of the components and their different spatial morphologies provide a means for separating the emission components. In this paper we apply the correlated component analysis method (CCA, Bonaldi et al. 2006; Ricciardi et al. 2010), which uses the spatial morphology of the components to perform the separation. The local Gould Belt system of current star formation is chosen as a particularly interesting area in which to make an accurate separation of the four foregrounds because of the different morphologies of the components. Gould (1879) first noted this concentration of prominent $\mathrm{OB}$ associations inclined at $20^{\circ}$ to the Galactic plane. It was next identified as an H I feature (Davies 1960; Lindblad 1967). Along with velocity data from $\mathrm{H} \mathrm{I}$ and CO combined with stellar distances from HIPPARCos, the total system appears to be a slowly expanding and rotating ring of gas and dust surrounding a system of OB stars within 500 pc of the Sun (Lindblad et al. 1997). A recent modelling of the Gould Belt system by Perrot \& Grenier (2003) gives semi-axes of $373 \times 233 \mathrm{pc}$ inclined at $17^{\circ}$ with an ascending node at $l=296^{\circ}$ and a centre $104 \mathrm{pc}$ distant from us lying at $l=180^{\circ}$. The Gould Belt thickness is $60 \mathrm{pc}$. The stars defining the system have ages younger than $30 \times 10^{6} \mathrm{yr}$.

The free-free emission from ionized hydrogen is wellunderstood (Dickinson et al. 2003). $\mathrm{H} \alpha$ is a good indicator of the emission measure in regions of low dust absorption. Elsewhere a correction has to be applied, which depends on where the absorbing dust lies relative to the $\mathrm{H} \alpha$ emission. The conversion of an emission measure value to a radio brightness temperature at a given frequency requires a knowledge of the electron temperature. Alternatively, an electron temperature can be derived by assuming a value for the dust absorption. Values for the electron temperature of $4000-8000 \mathrm{~K}$ are found in similar studies (Banday et al. 2003; Davies et al. 2006; Ghosh et al. 2012). Radio recombination-line observations on the Galactic plane (Alves et al. 2012) give values that agree with those of individual H II regions, having temperatures that rise with increasing distance from the Galactic centre; the value at the solar distance where the current study applies is 7000-8000 K.

The spectrum of synchrotron emission reflects the spectrum of the cosmic-ray electrons trapped in the Galactic magnetic field. At frequencies below a few $\mathrm{GHz}$ the brightness temperature spectral index, $\beta_{\mathrm{s}}$, is ranging from -2.5 to -2.7 (Broadbent et al. 1989). Between 1.0 GHz and WMAP and Planck frequencies, the spectral index steepens to values from -2.9 to -3.1 (Banday et al. 2003; Davies et al. 2006; Kogut et al. 2011).

Thermal dust dominates the Galactic emission at Planck frequencies above $100 \mathrm{GHz}$. The spectrum is well-defined here with temperature $T_{\mathrm{d}} \approx 18 \mathrm{~K}$ and spectral index $\beta_{\mathrm{d}}$ ranging from 1.5 to 1.8 (Planck Collaboration 2011c). In the frequency range 60-143 GHz the dust emission overlaps that of the free-free emission and AME, making it a critical range for component separation.

\footnotetext{
1 Planck (http://www.esa.int/Planck) is a project of the European Space Agency (ESA) with instruments provided by two scientific consortia funded by ESA member states (in particular the lead countries France and Italy), with contributions from NASA (USA) and telescope reflectors provided by a collaboration between ESA and a scientific consortium led and funded by Denmark.
}

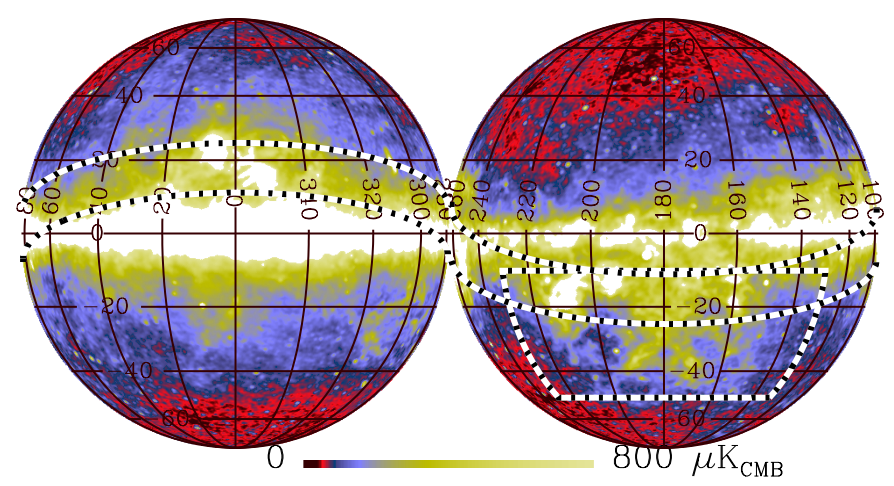

Fig. 1. Orthographic projection (looking towards the Galactic centre in the left panel and the Galactic anti-centre in the right panel) of the Planck CMB-subtracted $30 \mathrm{GHz}$ channel showing the Gould Belt and the region of interest for this paper (defined by $130^{\circ} \leq l \leq 230^{\circ}$ and $-50^{\circ} \leq b \leq-10^{\circ}$.

The AME component is highly correlated with the far infrared dust emission (Kogut 1996; Leitch et al. 1997; Banday et al. 2003; Lagache 2003; de Oliveira-Costa et al. 2004; Finkbeiner et al. 2004a; Davies et al. 2006; Dobler \& Finkbeiner 2008a; Miville-Deschênes et al. 2008; Ysard et al. 2010; Gold et al. 2011; Planck Collaboration 2011d) and is believed to be the result of electric dipole radiation from small spinning dust grains (Erickson 1957; Draine \& Lazarian 1998) in a range of environments (Ali-Haïmoud et al. 2009; Ysard \& Verstraete 2010). AME is seen in individual dust clouds associated with molecular clouds, photo-dissociation regions, reflection nebulae and H II regions (e.g., Finkbeiner et al. 2002, 2004b; Watson et al. 2005; Casassus et al. 2006, 2008; Dickinson et al. 2006, 2007, 2009; Scaife et al. 2007, 2010; AMI Consortium et al. 2009; Todorović et al. 2010; Murphy et al. 2010; Planck Collaboration 2011d; Dickinson 2013). In the present study we examine the AME spectrum in more extended regions.

\section{Definition of the region of interest and aim of the work}

The projection of the Gould Belt disc on the sky is a strip that is superimposed on the Galactic plane, except towards the Galactic centre (northern Gould Belt) and around $l=180^{\circ}$ (southern Gould Belt). In this work we consider the southern Gould Belt, which can be approximately defined by Galactic coordinates $130^{\circ} \leq l \leq 230^{\circ}$ and $-50^{\circ} \leq b \leq-10^{\circ}$ (see Fig. 1). This choice gives us a cleaner view of the Gould Belt, because the background emission from the Galactic plane is weaker here than towards the Galactic centre. Notable structures within the region are the Orion complex, Barnard's arc and the Taurus, Eridanus, and Perseus star-forming complexes. All these emitting regions, including the diffuse emission from the Eridanus shell at $-50^{\circ}<b<-30^{\circ}$, are at a distance within $500 \mathrm{pc}$ from us and thus they belong to the local inter-stellar medium (ISM) associated with the Gould Belt (e.g. Reynolds \& Ogden 1979; Boumis et al. 2001).

In Fig. 2 we show the CMB-subtracted Planck data at $1^{\circ}$ resolution, compared with the Haslam et al. (1982) $408 \mathrm{MHz}$ map, which mostly traces the synchrotron component, the Dickinson et al. (2003) $\mathrm{H} \alpha$ map, tracing free-free emission, and the $100 \mu \mathrm{m}$ map from Schlegel et al. (1998), tracing the dust emission. The visual inspection reveals dust-correlated features at low frequency, which could be attributed to AME. There is also prominent free-free emission, especially strong in the Barnard's arc 

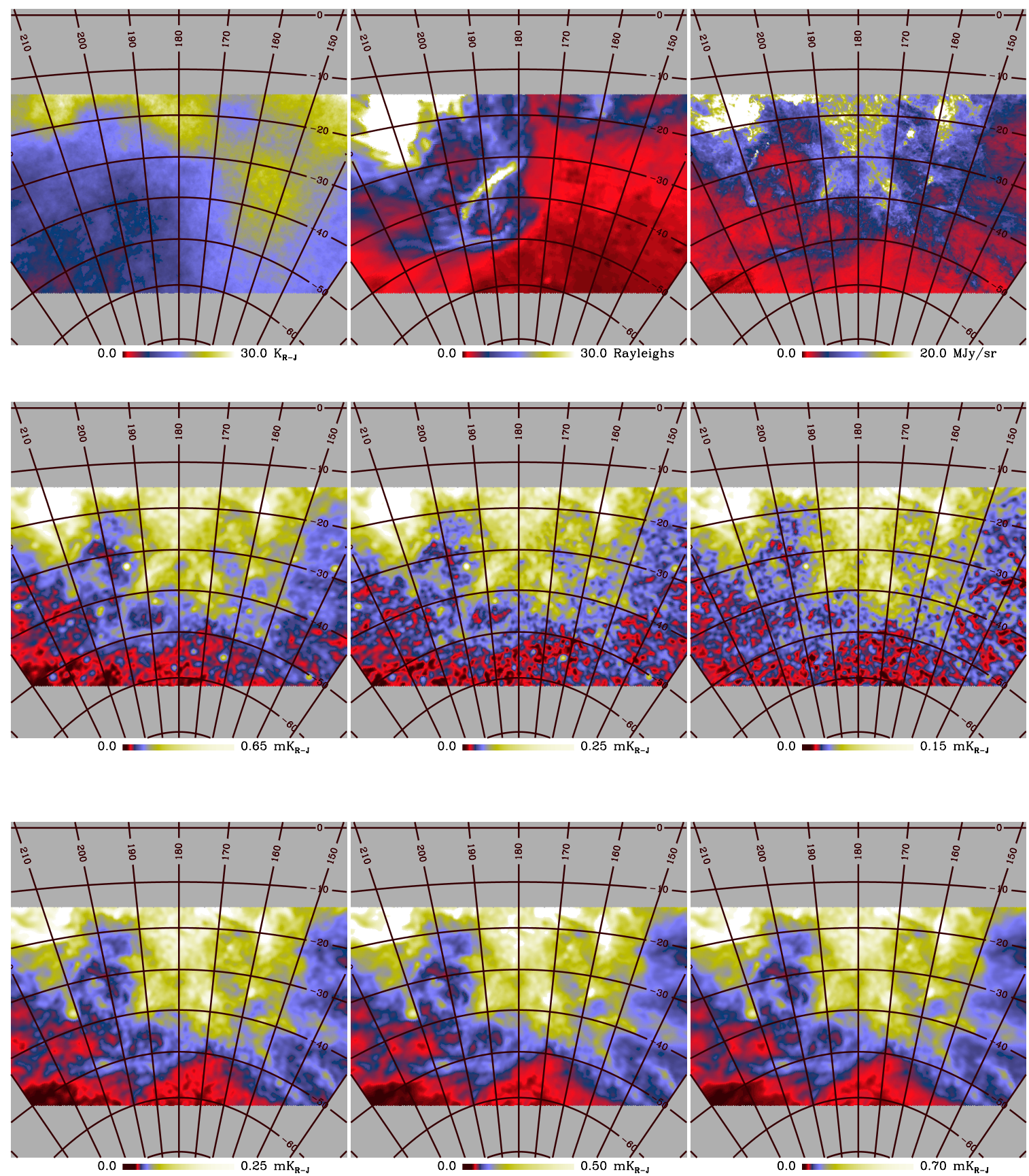

Fig. 2. Gnomonic projections of the region of interest. Top panels: Haslam et al. (1982) $408 \mathrm{MHz}$ map (left); H $\alpha$ map from Dickinson et al. (2003) (middle); and $100 \mu \mathrm{m}$ map from Schlegel et al. (1998) (right) at native resolution. Middle panels (left to right): Planck CMB-subtracted 30, 44, and $70 \mathrm{GHz}$ maps at $1^{\circ}$ resolution. Bottom panels (left to right): Planck CMB-subtracted 143,353 , and $857 \mathrm{GHz}$ at $1^{\circ}$ resolution.

region (towards $l=207^{\circ}, b=-18^{\circ}$ ). The synchrotron component appears to be sub-dominant with respect to the free-free emission and the AME.

This work aims at separating and studying the diffuse lowfrequency foregrounds, in particular AME and free-free emission, in the region of interest. This requires estimating the spectral behaviour of the AME (carried out in Sect. 4). We compare this spectrum with predictions for spinning dust emission, one of the mechanisms that is most often invoked to explain AME (Sect. 7). After reconstructing the free-free emission, we estimate the free-free electron temperature (Sect. 6), which relates free-free brightness to emission measure, and investigate the dependence of this result on the dust absorption fraction.

\section{Description of the analysis}

\subsection{Input data}

Planck (Tauber et al. 2010; Planck Collaboration 2011a) is the third-generation space mission that measures the anisotropy of the cosmic microwave background (CMB). It observes the sky in nine frequency bands covering $30-857 \mathrm{GHz}$ with high sensitivity and angular resolution from $31^{\prime}$ to $5^{\prime}$. The Low Frequency Instrument (LFI; Mandolesi et al. 2010; Bersanelli et al. 2010; Mennella et al. 2011) covers the 30, 44, and $70 \mathrm{GHz}$ bands with amplifiers cooled to $20 \mathrm{~K}$. The High Frequency Instrument (HFI; Lamarre et al. 2010; Planck HFI Core Team 2011a) covers the $100,143,217,353,545$, and $857 \mathrm{GHz}$ bands with 
Table 1. Summary of Planck data.

\begin{tabular}{|c|c|c|}
\hline $\begin{array}{l}\text { Central frequency } \\
{[\mathrm{GHz}]}\end{array}$ & Instrument & $\begin{array}{l}\text { Resolution } \\
\text { [arcmin] }\end{array}$ \\
\hline 28.5 & Planck LFI & 32.65 \\
\hline 44.1 & Planck LFI & $27 ! 92$ \\
\hline 70.3 & Planck LFI & 13.'01 \\
\hline 100 & Planck HFI & 9.'88 \\
\hline 143 & Planck HFI & $7 ! 18$ \\
\hline 217 & Planck HFI & 4.87 \\
\hline 353 & Planck HFI & 4.65 \\
\hline 545 & Planck HFI & 4.72 \\
\hline 857 & Planck HFI & $4 ! 39$ \\
\hline
\end{tabular}

bolometers cooled to $0.1 \mathrm{~K}$. Polarization is measured in all but the highest two bands (Leahy et al. 2010; Rosset et al. 2010). A combination of radiative cooling and three mechanical coolers produces the temperatures needed for the detectors and optics (Planck Collaboration 2011b). Two data-processing centres (DPCs) check and calibrate the data and make maps of the sky (Planck HFI Core Team 2011b; Zacchei et al. 2011). Planck's sensitivity, angular resolution, and frequency coverage make it a powerful instrument for galactic and extragalactic astrophysics as well as cosmology. Early astrophysics results are given in Planck Collaboration 2011c,d,e,f, 2013b, based on data taken between 13 August 2009 and 7 June 2010. Intermediate astrophysics results are now being presented in a series of papers based on data taken between 13 August 2009 and 27 November 2010.

The Planck data used throughout this paper are an internal data set known as DX7, whose properties are described in appendices to the LFI and HFI data processing papers (Planck Collaboration 2013a,b). However, we have tested the analysis to the extent that the results will not change if carried out on the maps that have been released to the public in March 2013.

The specifications of the Planck maps are reported in Table 1. The dataset used for the analysis consists of fullresolution frequency maps and the corresponding noise information. We indicate whenever the CMB-removed version of this dataset was used for display purposes.

When analysing the results, we applied a point source mask based on blind detection of sources above $5 \sigma$ in each Planck map, as described in Zacchei et al. (2011) and Planck HFI Core Team (2011b). Ancillary data were used throughout the paper for component-separation purposes, to simulate the sky and data, or to analyse our results. The full list of ancillary data is reported in Table 2 with the main specifications.

\subsection{Components}

The main diffuse components present in the data are CMB and Galactic synchrotron emission, free-free emission, thermal dust emission, and AME. The frequency spectrum of the CMB component is well-known: it is accurately described by a black-body with a temperature $T_{\mathrm{CMB}}=2.7255 \mathrm{~K}$ (Fixsen 2009).

Thermal dust emission dominates at high frequencies. Its spectral behaviour is a superposition of modified black-body components identified by temperature $T_{\text {dust }}$ and emissivity in$\operatorname{dex} \beta_{\mathrm{d}}$ :

$T_{\text {RJ,dust }}(v) \propto v^{\beta_{\mathrm{d}}+1} /\left[\exp \left(h v / k T_{\text {dust }}\right)-1\right]$,

where $k$ is the Boltzmann constant and $h$ is the Planck constant. In the approximation of a single component, over most of the sky we have $T_{\text {dust }} \approx 18 \mathrm{~K}$ and $\beta_{\mathrm{d}}$ of $1.5-1.8$ (Finkbeiner et al. 1999; Planck Collaboration 2011c,f).

The frequency spectrum of the free-free component is often described by a power-law with spectral index -2.14 in RJ units. A more accurate description (see, e.g. Planck Collaboration 2011d) is given by

$T_{\mathrm{RJ}, \mathrm{ff}}(v) \propto G(v) \times(v / 10)^{-2}$,

where $G=3.96\left(T_{4}\right)^{0.21}(v / 40)^{-0.14}$ is the Gaunt factor, which is responsible for the departure from a pure power-law behaviour. $T_{4}$ is the electron temperature $T_{\mathrm{e}}$ in units of $10^{4} \mathrm{~K}\left(T_{\mathrm{e}}\right.$ can range over $2000-20000 \mathrm{~K}$, but for most of the ISM it is 4000$15000 \mathrm{~K})$.

The spectral behaviour of synchrotron radiation can be described to first order by a power-law model with spectral index $\beta_{\mathrm{s}}$ that typically assumes values from -2.5 to -3.2 , depending on the position in the sky. Steepening of the synchrotron spectral index with frequency is expected due to energy losses of the electrons.

The frequency scaling of the AME component is the most poorly constrained. The distinctive feature is a peak around 20$40 \mathrm{GHz}$ (Draine \& Lazarian 1998; Dobler \& Finkbeiner 2008b; Dobler et al. 2009; Hoang et al. 2011). However, a power-law behaviour is compatible with most detections above $23 \mathrm{GHz}$ (Banday et al. 2003; Davies et al. 2006; Ghosh et al. 2012). This could be the result of a superposition of several peaked components along the line of sight or could indicate a peak frequency lower than $23 \mathrm{GHz}$. The most recent WMAP 9-yr results quote a peak frequency at low latitudes ranging from 10 to $20 \mathrm{GHz}$ for the spectrum in $\mathrm{K}_{\mathrm{R}-\mathrm{J}}$ units, which means $20-30 \mathrm{GHz}$ when considering flux density units.

\subsection{Component separation pipeline}

Several component-separation methods adopt the linear-mixture data model (see Appendix A for a full derivation). For each line of sight we write

$\boldsymbol{x}=\mathbf{H} \boldsymbol{s}+\boldsymbol{n}$,

where $\boldsymbol{x}$ and $\boldsymbol{n}$ contain the data and the noise signals. They are vectors of dimension $N_{\mathrm{d}}$, which is the number of frequency channels considered. The vector $\boldsymbol{s}$, with the dimension $N_{\mathrm{c}}$, contains the $N_{\mathrm{c}}$ unknown astrophysical components (e.g. CMB, dust emission, synchrotron emission, free-free emission, AME) and the $N_{\mathrm{d}} \times N_{\mathrm{c}}$ matrix $\mathbf{H}$, called the mixing matrix, contains the frequency scaling of the components for all frequencies. The elements of the mixing matrix are computed by integrating the source emission spectra within the instrumental bandpass. When working in the pixel domain, Eq. (3) holds under the assumption that the instrumental beam is the same for all frequency channels. In the general case, this is achieved by equalizing the resolution of the data maps to the lowest one. When working in the harmonic or Fourier domain, the convolution for the instrumental beam is a multiplication and is linearized without assuming a common resolution.

Within the linear model, we can obtain an estimate $\hat{s}$ of the components $s$ through a linear mixture of the data:

$\hat{s}=\mathbf{W} \boldsymbol{x}$

where $\mathbf{W}$ is called the reconstruction matrix. Suitable reconstruction matrices can be obtained from the mixing matrix $\mathbf{H}$. For example,

$\mathbf{W}=\left[\mathbf{H}^{\mathrm{T}} \mathbf{C}_{\mathrm{n}}^{-1} \mathbf{H}\right]^{-1} \mathbf{H}^{\mathrm{T}} \mathbf{C}_{\mathrm{n}}^{-1}$ 
Table 2. Summary of ancillary data.

\begin{tabular}{lccc}
\hline \hline $\begin{array}{l}\text { Central frequency } \\
{[\mathrm{GHz}]}\end{array}$ & Label & $\begin{array}{c}\text { Resolution } \\
{[\operatorname{arcmin}]}\end{array}$ & Reference \\
\hline 0.408 & Haslam & 60 & Haslam et al. (1982) \\
& H $\alpha$ & 60 & Dickinson et al. (2003) \\
H $\alpha$ & $6-60$ & Finkbeiner (2003) \\
$94.8-94$ & WMAP 7-yr & $56.8-13.8$ & Jarosik et al. (2011) \\
2997 & & 60 & Finkbeiner et al. (1999) \\
24983,2997 & $100 \mu \mathrm{m}$ & 5 & Schlegel et al. (1998) \\
& IRIS Band 1, 4 & 4 & Miville-Deschênes \& Lagache (2006) \\
& $E(B-V)$ & 5 & Schlegel et al. (1998) \\
\hline
\end{tabular}

is called the generalized least-squares (GLS) solution and only depends on the mixing matrix and on the noise covariance $\mathbf{C}_{n}$.

The mixing matrix is the key ingredient of component separation. However, as discussed in Sect. 3.2, the frequency spectra of the components are not known with sufficient precision to perform an accurate separation. To overcome this problem, our component-separation pipeline implements a first step in which the mixing matrix is estimated from the data and a second one in which this result is exploited to reconstruct the amplitudes of the components.

\subsubsection{Estimating the mixing matrix}

To estimate the mixing matrix we relied on the CCA (Bonaldi et al. 2006; Ricciardi et al. 2010), which exploits second-order statistics of the data to estimate the frequency scaling of the components on defined regions of the sky (sky patches). We used the harmonic-domain version of the CCA, whose basic principles of operation are reported in Appendix A. This code works on square sky patches using Fourier transforms. It exploits the data auto- and cross-spectra to estimate a set of parameters describing the frequency scaling of the components. The patch-by-patch estimation prevents the detection of small-scale spatial variations of the spectral properties. On the other hand, by using a large number of samples we retain more information, which provides good constraints, even when the components have similar spectral behaviour. The CCA has been successfully used to separate the synchrotron, free-free and AME components from WMAP data in Bonaldi et al. (2007).

We used a patch size of $20^{\circ} \times 20^{\circ}$, obtained as a trade-off between having enough statistics for a robust computation of the data cross-spectra and limited spatial variability of the foreground properties. Given the dimension of the region of interest, we have ten independent sky patches. However, exploiting a redundant number of patches, widely overlapping each other, enables us to eradicate the gaps between them and obtain a result that is independent of any specific selection of patches. We covered the region of interest with patches spaced by $2^{\circ}$ in both latitude and longitude. By re-projecting the results of the CCA on a sphere and averaging the outputs for each line of sight, we can synthesize smooth, spatially varying maps of the spectral parameters (see Ricciardi et al. 2010 for more details).

\subsubsection{Reconstruction of the component amplitudes}

The amplitudes were reconstructed in pixel space at $1^{\circ}$ resolution using Eqs. (4) and (5), exploiting the output of the previous step. To equalize the resolution of the data maps, the $a_{\ell m}$ of each map were multiplied by a window function, $W_{\mathrm{S}}^{(\ell)}$, given by a $1^{\circ}$ Gaussian beam divided by the instrumental beam of the corresponding channel (assumed to be Gaussian with a full width half maximum (FWHM) as specified in Table 1). This corresponds, in real space, to a convolution with a beam $B_{\mathrm{S}}$. To obtain an estimate of the corresponding noise after smoothing, the noise variance maps should be convolved with $B_{\mathrm{N}}=\left(B_{\mathrm{S}}\right)^{2}$. We did this again in harmonic-space, after obtaining the window function $W_{\mathrm{N}}^{(\ell)}$, corresponding to $B_{\mathrm{N}}$, by Legendre-transforming $W_{\mathrm{S}}^{(\ell)}$, squaring the result, and Legendre-transforming back.

The smoothing process also correlates noise between different pixels, which means that the rms per pixel obtained as detailed above is not a complete description of the noise properties. However, estimating of the full covariance of noise (and its propagation through the separation in Eqs. (4) and (5)) is very computationally demanding. In this work we took into account only the diagonal noise covariance and neglected any correlation between noise in different pixels. In a signal-dominated case, such as the one considered here, the errors on the noise model have only a weak impact on the results.

\section{AME frequency spectrum}

We modelled the mixing matrix to account for five components: the $\mathrm{CMB}$, the synchrotron emission, the thermal dust emission, the free-free emission, and the AME. We neglected the CO component by excluding the 100 and $217 \mathrm{GHz}$ Planck channels from the analysis, which are significantly contaminated by the $\mathrm{CO}$ lines $J=1 \rightarrow 0$ and $J=2 \rightarrow 1$, respectively (Planck HFI Core Team 2011b). CO is also present at $353 \mathrm{GHz}$, where it can contaminate the dust emission by up to $3 \%$ in the region of interest, and at 545 and $857 \mathrm{GHz}$, where the contamination is negligible. To estimate the mixing matrix we used the following datasets

- the Planck 30, 44, 70, 143 and $353 \mathrm{GHz}$ channels,

- the WMAP 7-yr K band (23 GHz),

- the Haslam et al. $408 \mathrm{MHz}$ map,

- the predicted free-free emission at $23 \mathrm{GHz}$ based on the $\mathrm{H} \alpha$ Dickinson et al. (2003) template corrected for dust absorption with the Schlegel et al. (1998) $E(B-V)$ map by assuming a dust absorption fraction of 0.33 .

We verified that including the WMAP Ka-W bands in this analysis did not produce appreciable changes in the results. The explored frequency range is now covered by Planck data with higher angular resolution and sensitivity. Caution is needed when using $\mathrm{H} \alpha$ as a free-free tracer: dust absorption (Dickinson et al. 2003) and scattering of $\mathrm{H} \alpha$ photons from dust grains (Wood \& Reynolds 1999; Dong \& Draine 2011) cause dust-correlated errors in the free-free template, which could bias the AME spectrum. The impact of such biases was assessed through simulations as described in Sect. 4.1. 
For dust emission we used the model of Eq. (1) with $T_{\mathrm{d}}=$ $18 \mathrm{~K}$ and estimated the dust spectral index $\beta_{\mathrm{d}}$. The reason why we fixed the dust temperature is that this parameter is mostly constrained by high-frequency data, which we do not include in this analysis. In fact, a single modified black-body model with constant $\beta_{\mathrm{d}}$ only poorly describes the dust spectrum across the frequency range covered by Planck. In particular, $\beta_{\mathrm{d}}$ is derived to be flatter in the microwave $(v \leq 353 \mathrm{GHz})$ than in the millimetre range $(v>353 \mathrm{GHz})$.

The temperature $T_{\mathrm{d}}=18 \mathrm{~K}$ we adopted is consistent with the one-component dust model by Finkbeiner et al. (1999) and agrees well with the median temperature of $17.7 \mathrm{~K}$ estimated at $|b|>10^{\circ}$ by Planck Collaboration (2011c). For the dust spectral index we obtained $\beta_{\mathrm{d}}=1.73 \pm 0.09$. For synchrotron radiation we adopted a power-law model with a fixed spectral in$\operatorname{dex} \beta_{\mathrm{s}}=-2.9$ (e.g., Miville-Deschênes et al. 2008), because the weakness of the signal prevented a good estimation of this parameter. We verified that different choices for $\beta_{\mathrm{s}}$ (up to a $10 \%$ variation, $\beta_{\mathrm{s}}$ from -2.6 to -3.2 ) changed the results for the other parameters only by about $1 \%$, due to the weakness of the synchrotron component with respect to the AME and thermal dust. As a spectral model for the AME we adopted the best-fit model of Bonaldi et al. (2007), which is a parabola in the $\log (S)-\log (v)$ plane parametrized in terms of peak frequency $v_{\mathrm{p}}{ }^{2}$ and slope at $60 \mathrm{GHz} m_{60}$ :

$\log T_{\mathrm{RJ}, \mathrm{AME}}(v) \propto\left(\frac{m_{60} \log v_{\mathrm{p}}}{\log \left(v_{\mathrm{p}} / 60\right)}+2\right) \log v+\frac{m_{60}(\log v)^{2}}{2 \log \left(v_{\mathrm{p}} / 60\right)}$.

Details of the model and justification of this choice are given in Appendix B. We also tested a pure power-law model $\left(T_{\mathrm{RJ}, \mathrm{AME}}(v) \propto v^{\alpha}\right)$ for AME, fitting for the spectral index $\alpha$, but we were unable to obtain valid estimates in this case. This is what we expect when the true spectrum presents some curvature, as verified through simulations (see Sect. 4.1 and Appendix C).

Our results for the AME spectrum are shown in the left panels of Fig. 3. On average, the AME peaks at $25.5 \mathrm{GHz}$, with a standard deviation of $0.6 \mathrm{GHz}$, which is within estimation errors $(1.5 \mathrm{GHz})$. This means we find no significant spatial variations of the spectrum of the AME in the region of the sky considered here. However, we recall that this only applies to diffuse AME, because our pipeline cannot detect small-scale spatial variations, and we are restricted to a limited area of the sky.

Our results on the peak frequency of the AME are similar to those of Planck Collaboration (2011d) for Perseus and $\rho$ Ophiuchi. The WMAP 9-yr MEM analysis (Bennett et al. 2012) measures the position of the peak for the spectrum in $\mathrm{K}_{\mathrm{R}-\mathrm{J}}$ units and finds a typical value of $14.4 \mathrm{GHz}$ for diffuse AME at low latitudes, which roughly corresponds to $27 \mathrm{GHz}$ when the spectrum is in flux density units. According to previous work, at higher latitudes the peak frequency is probably lower (see e.g. Banday et al. 2003; Davies et al. 2006; Ghosh et al. 2012). Interestingly, the same CCA method used in this paper yields $v_{\mathrm{p}}$ around $22 \mathrm{GHz}$ when applied to the North Celestial Pole region (towards $l=125^{\circ}, b=25^{\circ}$, Bonaldi \& Ricciardi 2012). Spatial variations of the physical properties of the medium could explain these differences.

In the hypothesis of spinning dust emission, there are many ways to achieve a shift in the peak frequency. Because the available data do not allow us to distinguish between them, we just mention two main possibilities. The first is a change in the density of the medium, lower densities being associated with lower

\footnotetext{
2 The peak frequency $v_{\mathrm{p}}$ is defined for the specrum in flux density units.
}

peak frequencies (see also Table B.1). Indeed, the AME spectrum is modelled with densities of $0.2-0.4 \mathrm{~cm}^{-3}$ in Bonaldi \& Ricciardi (2012), while it requires higher densities in the Gould Belt region, as discussed in Sect. 7. The second possibility is a change in the size distribution of the dust grains, smaller sizes yielding higher peak frequencies. We return to these aspects in Sect. 7.

\subsection{Assessment through simulations}

The reliability of our results has been tested with simulations. The main purposes of this assessment are

- to verify the ability of our procedure to accurately recover the AME spectrum for different input models and

- to investigate how the use of foreground templates - free-free in particular - can bias the results.

We assessed this by applying the procedure described in Sect. 4 to sets of simulated data, whose true inputs are known. For the first target, we performed three separate simulations including a different AME model: two spinning-dust models, peaking at $19 \mathrm{GHz}$ and $26 \mathrm{GHz}$, and a spatially varying power-law. For the second target, we introduced dust-correlated biases in the freefree template and quantified their impact on the estimated parameters. The full description of the simulations and of the tests performed is given in Appendix C.

The results are displayed in the right panels of Fig. 3. In the top panel we show the true spectrum (solid line) and the estimated spectrum with errors (shaded area) for each of the three tested input models. The red and blue areas distinguish between two free-free templates (referred to as $\mathrm{FF}_{1}$ and $\mathrm{FF}_{2}$ ), which are biased in a different way with respect to the simulated free-free component. In the middle and bottom panels we show the histograms of the recovered spectral parameters compared with the true inputs (vertical lines); the red and blue colours are as before. We conclude the following:

- If the input AME is a convex spectrum, we are able to accurately recover the peak frequency, $v_{\mathrm{p}}$, for both the 19 and $26 \mathrm{GHz}$ input values. Our pipeline is able to distinguish very clearly between the two input models; biases in the free-free template do not affect the recovery of the peak frequency.

- The estimated spectrum can be slightly biased above 40$50 \mathrm{GHz}$, where the AME is faint, as a result of limitations of the spectral model we used (see Appendix B) and errors in the free-free template. The systematic error on $m_{60}$ is quantified as 0.5-0.6.

- If the input AME spectrum is a power-law, we obtain a good recovery when fitting for a spectral index.

When the AME is a power-law, the parabolic model is clearly wrong, because the parameter describing the position of the peak is completely unconstrained and the model steepens considerably with frequency. Similarly, when the AME is a curved spectrum, the power-law model is too inaccurate to describe it. As expected, both these estimates fail to converge. We note that the distribution of $m_{60}$ recovered on real data is quite different from that obtained from the simulation. This could indicate spatial variability of the true spectrum, which is not included in the simulation. It could also indicate that the systematic errors on $m_{60}$ predicted by simulations, as we just described, are different in different regions of the sky, thus creating a non-uniform effect. 
Planck Collaboration: Galactic diffuse components in the Gould Belt system
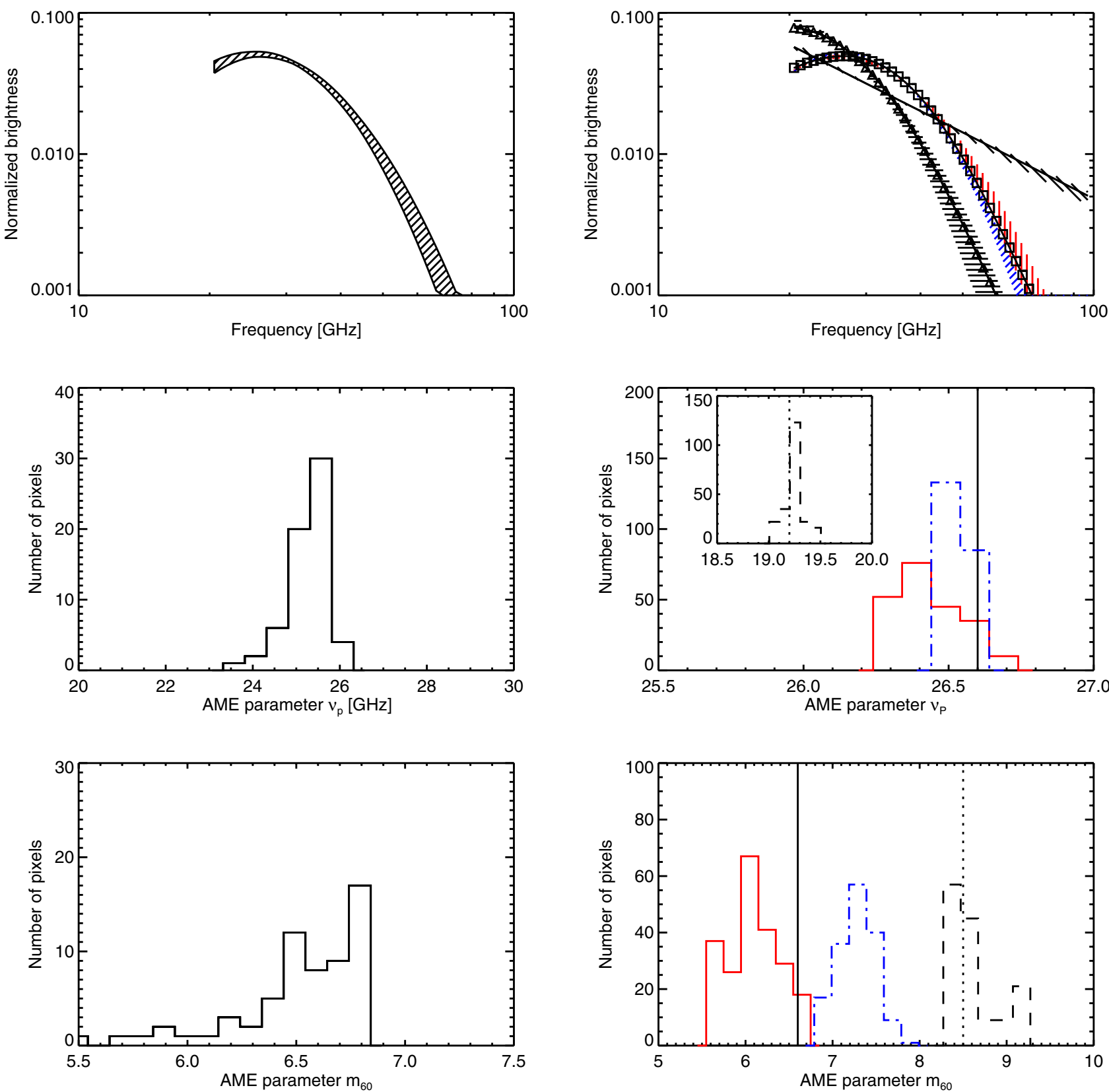

Fig. 3. CCA estimates of the AME frequency spectrum in the region of interest for real data (left panels) and simulated data (right panels). Top: estimated spectra including $1 \sigma$ errors. Middle and bottom: histograms of the spectral parameters $m_{60}$ and $v_{\mathrm{p}}$ on $N_{\text {side }}=16$ estimated spectral index maps. For the simulated case (right panels) we considered two convex spectra peaking at $19 \mathrm{GHz}$ and $26 \mathrm{GHz}$ and a power-law model. Top right: the true inputs are shown as solid black lines (power-law) with triangles (19 GHz peak) and squares (26 GHz peak) and the estimates as shaded areas. The blue and red colours show estimates derived by exploiting the free-free templates $\mathrm{FF}_{1}$ and $\mathrm{FF}_{2}$ described in Appendix C. Middle and bottom-right panels: the true inputs are shown as solid and dotted vertical lines for the simulations peaking at $26 \mathrm{GHz}$ and $19 \mathrm{GHz}$ respectively; the blue dot-dashed and red solid histograms show the estimates obtained using the $\mathrm{FF}_{1}$ and $\mathrm{FF}_{2}$ templates, and the black dashed lines show the estimates for the $19 \mathrm{GHz}$ input spectrum.

\section{Reconstruction of the amplitudes}

We reconstructed the amplitude of the components on the $1^{\circ}$ resolution version of the dataset. We used the same frequencies as for estimating the mixing matrix, except for the free-free template, which was excluded to avoid possible biases in the reconstruction. The results are shown in Fig. 4. The first and second rows show the components reconstructed at $30 \mathrm{GHz}$ (from left to right: synchrotron emission, free-free emission, AME, and thermal dust emission) and the corresponding noise rms maps.
Thanks to the linearity of the problem, the noise variance maps can be obtained by combining the noise variance maps of the channels at $1^{\circ}$ degree resolution with the squared reconstruction matrix $\mathbf{W}$. The noise on the synchrotron and thermal dust maps is low compared with that for free-free and AME. This is because the $408 \mathrm{MHz}$ map and the Planck $353 \mathrm{GHz}$ channel constrain well the amplitudes of synchrotron and thermal dust emission.

The AME component is correlated at about $60 \%$ and $70 \%$ with the $100 \mu \mathrm{m}$ and the $E(B-V)$ dust templates by Schlegel et al. (1998), at about $40 \%$ with Haslam et al. (1982) 

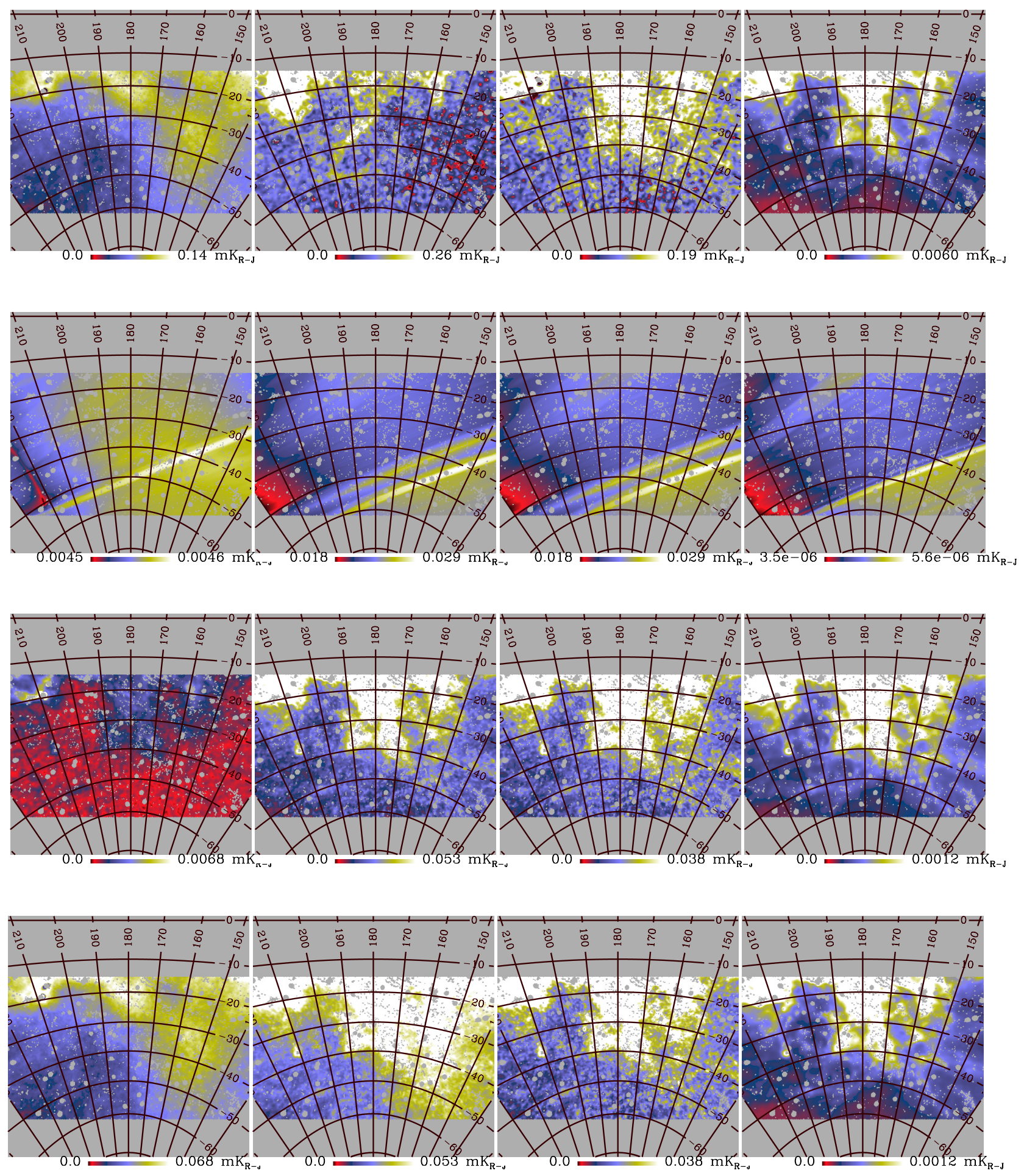

Fig. 4. $1^{\circ}$ resolution reconstruction at $30 \mathrm{GHz}$ of (from left to right): synchrotron emission; free-free emission; AME; and thermal dust emission. These reconstructions are performed as described in Sect. 3.3.2. Rows from top to bottom: component amplitudes; noise rms; predicted rms of component separation error due to the estimation of AME and thermal dust spectra; and predicted rms of component separation error including a random error on $\beta_{\mathrm{s}}=-2.9 \pm 0.1$.

$408 \mathrm{MHz}$ and at about $20 \%$ with $\mathrm{H} \alpha$. This favours emission mechanisms based on dust over other hypotheses, such as curved synchrotron emission and free-free emission. The $E(B-V)$ template correlates better with thermal dust emission than the $100 \mu \mathrm{m}$ map (the correlation coefficients are $0.73 \pm 0.01$ and $0.96 \pm 0.01$, respectively). This is expected if AME is dust emission. In fact, both spinning dust and thermal dust emission are proportional to the column density, for which $E(B-V)$ is a better estimator than the $100 \mu \mathrm{m}$ emission, which is strongly affected by the dust temperature.

The errors due to the separation process (third and fourth row of Fig. 4) are obtained by propagating via a Monte-Carlo simulation the uncertainties on the mixing matrix estimated by CCA to the reconstruction of the components (see Ricciardi et al. 2010, 

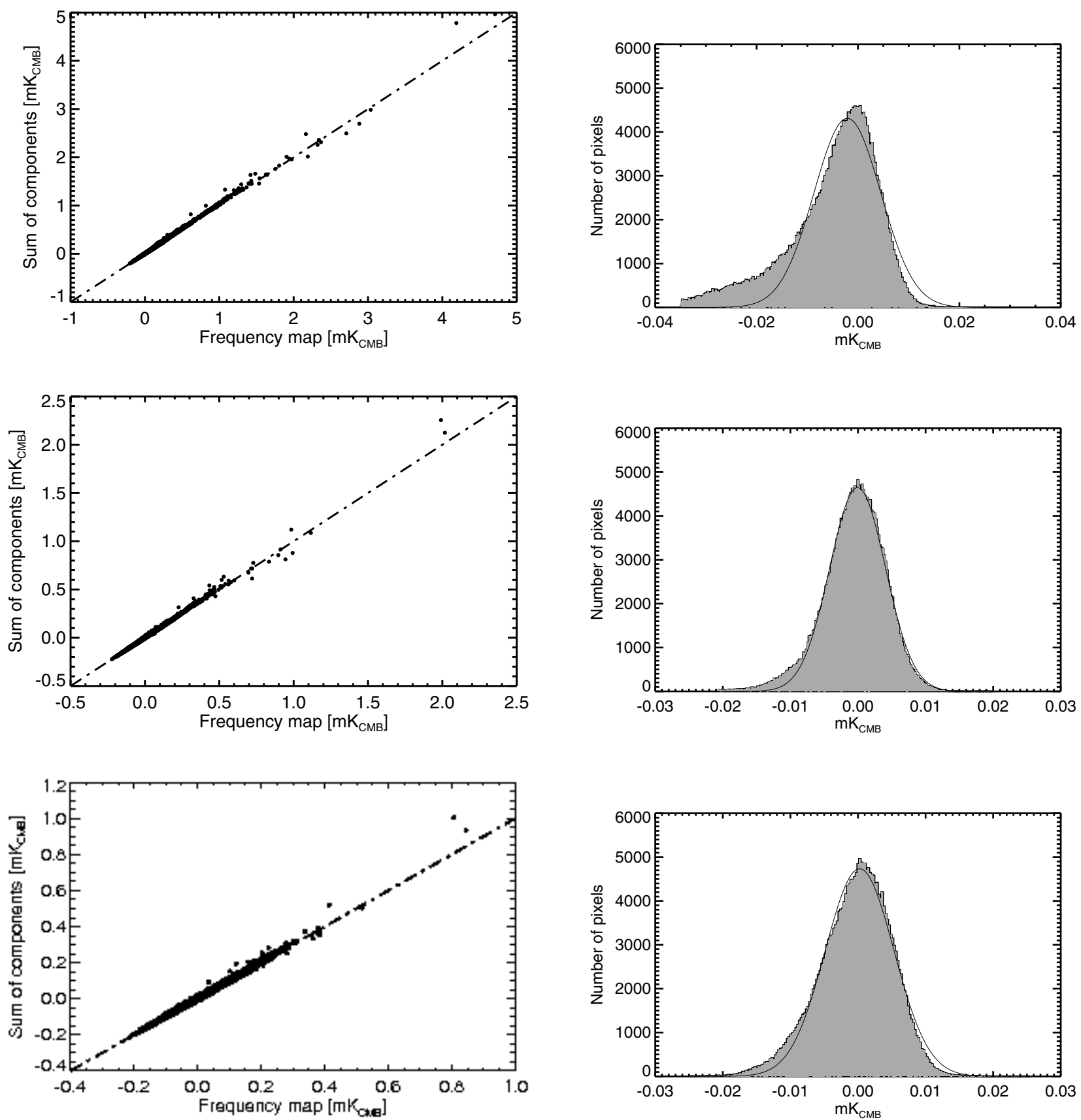

Fig. 5. Validation of the reconstructed components shown in Fig. 4. Left panels: sum of the components vs frequency maps at 30, 44 , and $70 \mathrm{GHz}$ (from top to bottom). The line is the $x=y$ relation. Right panels: pixel distribution of the residual (frequency map-sum of the components) maps compared with the best-fit Gaussian distribution.

for more details). Essentially, the mixing matrix parameters are randomized according to their posterior distributions; the component separation error on the amplitudes is estimated to be the variance of GLS reconstructions for different input mixing matrices.

One complication is that we did not estimate the synchrotron spectral index, but fixed it at $\beta_{\mathrm{s}}=-2.9$. Thus, we do not have errors on the synchrotron spectral index from our analysis. We therefore considered two cases: one in which we propagated only the errors on the AME and thermal dust spectral parameters, thus assuming no error on $\beta_{\mathrm{s}}$ (third row of Fig. 4), and another in which we included an indicative random error $\Delta \beta_{\mathrm{s}}=0.1$ (last row of Fig. 4).

The predicted error due to separation is generally higher than noise and on average of about $15-20 \%$ of the component amplitude for AME, free-free, and dust emission. Once we allow some scatter on $\beta_{\mathrm{s}}$, the predicted error on synchrotron emission becomes about 50\%: this indicates that the reconstruction of this component is essentially prior-driven. The inclusion of $\Delta \beta_{\mathrm{s}}$ has some effect on the error prediction for free-free emission, while AME and dust emission are mostly unaffected.

To evaluate the quality of the separation we compared the frequency maps with the sum of the reconstructed components at the same frequency. In the left panels of Fig. 5 we plot the sum of the components for 30,44 , and $70 \mathrm{GHz}$ against the amplitude of the frequency map. The comparison is made at $1^{\circ}$ resolution with $N_{\text {side }}=128$ pixels. The dashed line indicates the $x=y$ relation, which corresponds to the ideal case in which the two maps are identical. 

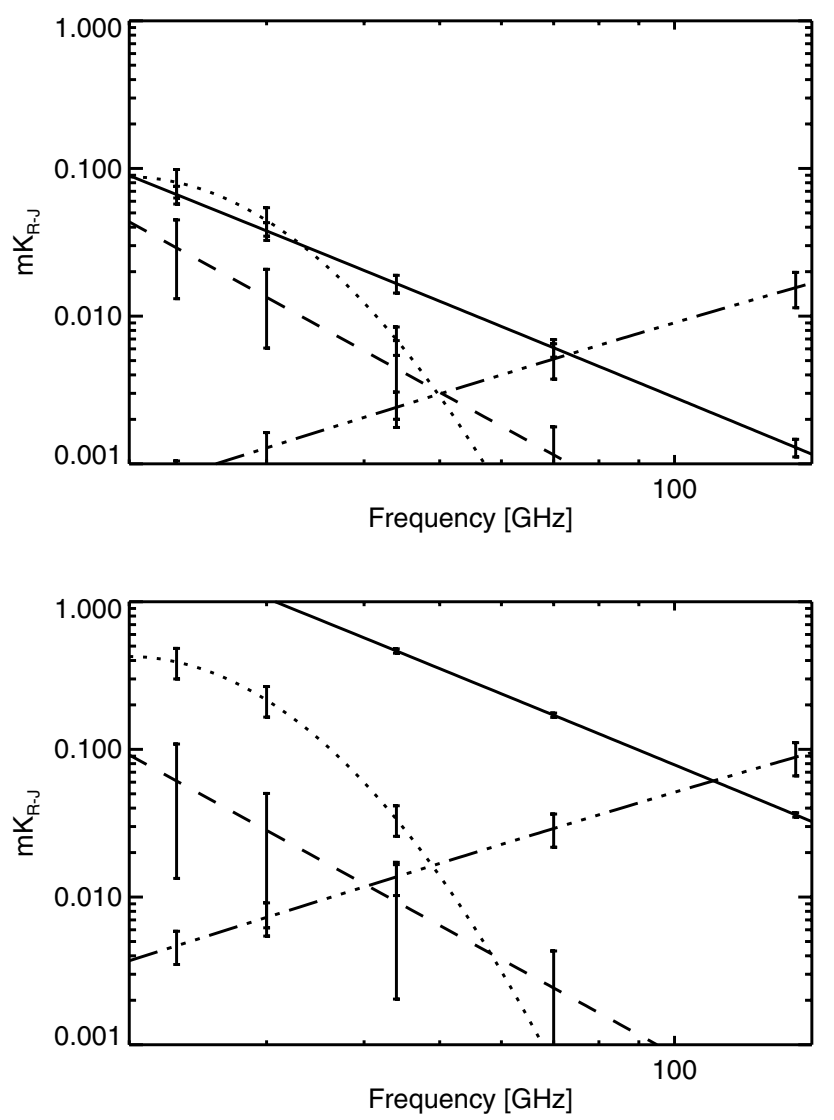

Fig. 6. Estimated spectra of synchrotron emission (dashed line), freefree emission (solid line), thermal dust emission (dash-dotted line), and AME (dotted line) for average local properties (top) and for Barnard's region (bottom).

The agreement between data and predictions is in general very good. The scatter of the points does not measure the quality of the separation, but the signal-to-noise ratio of the maps. It increases from 30 to $70 \mathrm{GHz}$, as the foreground signal becomes weaker. The errors in the component separation show up as systematic departures of the data from the prediction. Because those are not apparent, we also show on the right panels of Fig. 5 the pixel distribution of the residual map compared with the bestfit Gaussian distribution. At 44 and $70 \mathrm{GHz}$ the scatter, though quite small, dominates the residual and covers the systematic effects, with the exception of a few outliers, mostly due to compact sources. At $30 \mathrm{GHz}$ the scatter is low enough to reveal a feature: a sub-sample of pixels in which the reconstructed signal is higher than the true one, thus creating a negative in the residual.

This kind of systematic effect is very difficult to avoid when separating many bright components, because small errors in the mixing matrix cause bright features in the residual maps. Our Monte Carlo approach is able to propagate these errors however. At $30 \mathrm{GHz}$ the brightest components are AME and free-free emission, for which the predicted component separation error is on average $0.04-0.05 \mathrm{mK}_{\mathrm{CMB}}$, in agreement with the level of the non-Gaussian residuals. Coherent structures in the residual maps are induced by the low resolution of the maps of spectral parameters, which means that over nearby pixels the error in the mixing matrix, and thus on the separation, is similar.

In Fig. 6 we show the amplitude of the components as a function of frequency. The top panel represents the typical behaviour in the Gould Belt, while the bottom one refers to a particular case, Barnard's region, where free-free emission is particularly strong. The points are the average amplitude of the components at each frequency within the selected regions of the sky. The scaling of the amplitudes with frequency is, by construction, given by the spectral model estimated with CCA. The error bars measure the scatter induced on the amplitudes by the errors on the spectral parameters (also including $\Delta \beta_{\mathrm{s}}=0.1$ ).

\section{Free-free electron temperature}

The intensity of the free-free emission at a given frequency with respect to $\mathrm{H} \alpha$ can be expressed as

$$
\frac{T_{\mathrm{ff}}(v)\left[\mu \mathrm{K}_{\mathrm{R}-\mathrm{J}}\right]}{\mathrm{H} \alpha[\text { Rayleighs }]}=14 T_{4}^{0.517} \times 10^{0.029 / T_{4}} \times 1.08 G(v)(v / 10)^{-2},
$$

where $\mathrm{G}(v)$ is the Gaunt factor already introduced in Sect. 3.2 and $T_{4}$ is the electron temperature $T_{\mathrm{e}}$ in units of $10^{4} \mathrm{~K}$. In the previous equation, $\mathrm{H} \alpha$ has been corrected for dust absorption. Following Dickinson et al. (2003), the correction depends on $f_{\mathrm{d}}$, the effective dust fraction in the line of sight that absorbs the $\mathrm{H} \alpha$. Therefore $f_{\mathrm{d}}$ and $T_{\mathrm{e}}$ are degenerate parameters.

The ratio $T_{\mathrm{ff}}(v) / \mathrm{H} \alpha$ can be obtained by comparing the $\mathrm{H} \alpha$ and free-free emission from component separation through a temperature-temperature plot (T-T analysis). We made free-free versus $\mathrm{H} \alpha$ plots by using the CCA free-free solution at $30 \mathrm{GHz}$ and both the Dickinson et al. (2003) and the Finkbeiner (2003) $\mathrm{H} \alpha$ templates corrected for dust absorption for different values of $f_{\mathrm{d}}$. We considered $3^{\circ}$ resolution maps, sampled with $N_{\text {side }}=64$ pixels. In addition to point sources, we excluded from the analysis the region most affected by dust absorption based on the Schlegel et al. (1998) $E(B-V)$ map, as shown in the top panel of Fig. 7. The electron temperature $T_{\mathrm{e}}$ was inferred by fitting the data points with a linear relation and converting the best-fit slope to $T_{\mathrm{e}}$ through Eq. (7). The error on $T_{\mathrm{e}}$ was derived from the error on the best-fit slope given by the fitting procedure through error propagation. In the bottom panel of Fig. 7 we show the T-T plots for the Dickinson et al. (2003) $\mathrm{H} \alpha$ template corrected for $f_{\mathrm{d}}=0.3$ (red points), and the best-fit linear relations to the T-T plots for different values of $f_{\mathrm{d}}=0.3$ (lines). The electron temperatures are reported in the top part of Table 3. We obtain $T_{\mathrm{e}}=5900-3900 \mathrm{~K}$ with $f_{\mathrm{d}}=0-0.5$ for the Dickinson template; the Finkbeiner template yields generally higher, but consistent, values $\left(T_{\mathrm{e}}=5800-4300 \mathrm{~K}\right.$ with $\left.f_{\mathrm{d}}=0-0.5\right)$.

\subsection{Comparison of the cross-correlation with templates}

An alternative way of computing $T_{\mathrm{ff}}(v) / \mathrm{H} \alpha$ and $T_{\mathrm{e}}$ is through cross-correlation of the $\mathrm{H} \alpha$ template with frequency maps (C-C analysis). We simultaneously cross-correlated the templates for free-free, dust, and synchrotron emission, as described in Ghosh et al. (2012). We used the $408 \mathrm{MHz}$ map from Haslam et al. (1982) as a tracer of synchrotron emission, the Dickinson et al. (2003) $\mathrm{H} \alpha$ as a tracer of free-free emission, and the Finkbeiner et al. (1999) model eight $94 \mathrm{GHz}$ prediction as a tracer of dust emission. We used the same resolution, pixel size, and sky mask as adopted for the T-T analysis $\left(3^{\circ}\right.$ and $\left.N_{\text {side }}=64\right)$. As pointed out by Ghosh et al. (2012), at this resolution the template-fitting analysis is more reliable than at $1^{\circ}$ because the smoothing reduces artefacts in the templates. The correlation coefficients were computed for each emission process at a given frequency by minimizing the generalized $\chi^{2}$ expression. We also fitted for an additional monopole term that can account for offset contributions in all templates and the data in a way that does not 
Planck Collaboration: Galactic diffuse components in the Gould Belt system

Table 3. Inferred $T_{\mathrm{e}}[\mathrm{K}]$ for the T-T and $\mathrm{C}-\mathrm{C}$ analysis using different $\mathrm{H} \alpha$ templates and dust absorption fractions $f_{\mathrm{d}}$.

\begin{tabular}{lccccc}
\hline \hline Method & Template & $f_{\mathrm{d}}=0.0$ & $f_{\mathrm{d}}=0.1$ & $f_{\mathrm{d}}=0.3$ & $f_{\mathrm{d}}=0.5$ \\
\hline T-T analysis & Dickinson & $5900 \pm 1200$ & $5400 \pm 1000$ & $4600 \pm 1200$ & $3900 \pm 1200$ \\
& Finkbeiner & $5800 \pm 1400$ & $5400 \pm 1200$ & $4700 \pm 1200$ & $4300 \pm 800$ \\
C-C analysis & Dickinson & $5300 \pm 1500$ & $4500 \pm 1400$ & $3100 \pm 1100$ & $2400 \pm 1000$ \\
& Finkbeiner & $7000 \pm 1700$ & $6500 \pm 1500$ & $5200 \pm 1300$ & $3800 \pm 1100$ \\
\hline
\end{tabular}
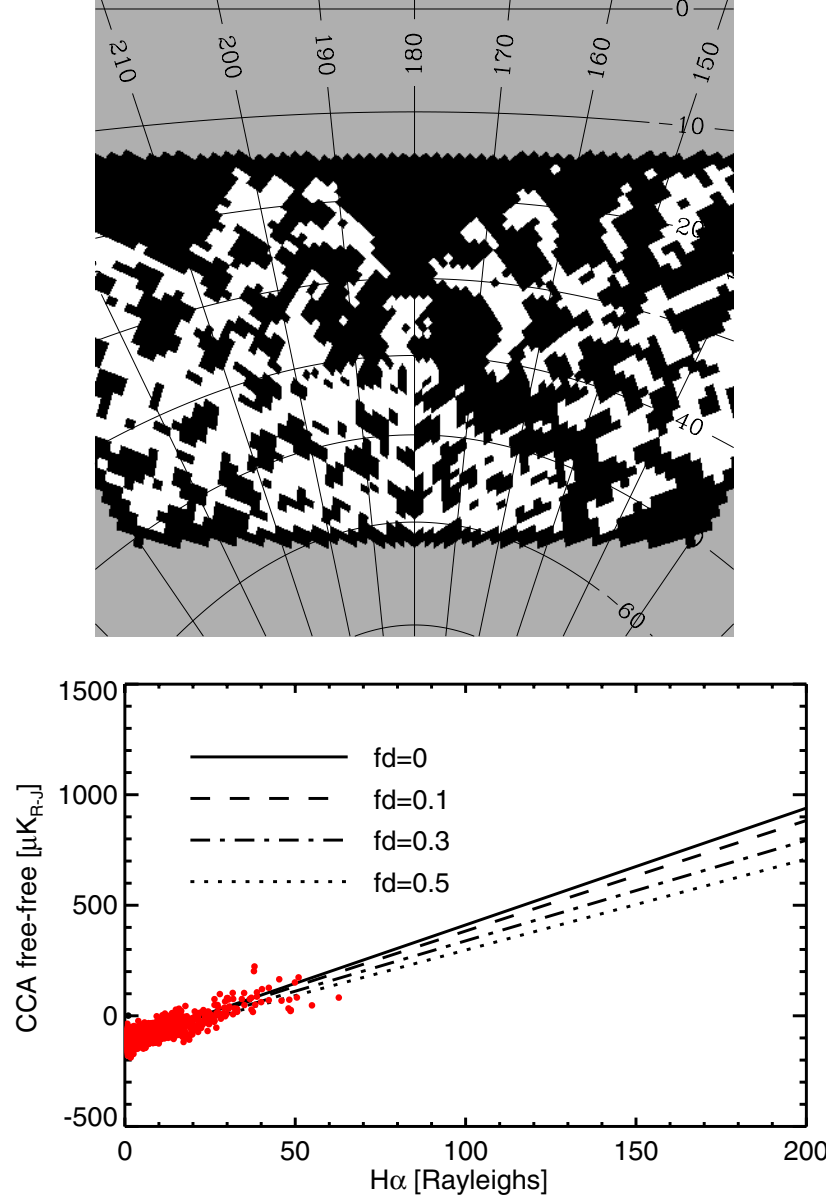

Fig. 7. T-T analysis for the estimation of $T_{\mathrm{e}}$. Top: gnomonic projection showing the mask used (masked pixels are in black, while pixels used in the analysis are in white). Bottom: T-T plot comparing the CCA freefree solution with the $\mathrm{H} \alpha$ template for $f_{\mathrm{d}}=0.3$ (points) and linear fits to the T-T plots for different values of $f_{\mathrm{d}}$ (lines).

bias the results (Macellari et al. 2011). The chance correlation of the templates with the CMB component in the data causes a systematic error in the correlated coefficients and was estimated using simulations. We generated 1000 random realizations of the CMB using the WMAP best-fit $\Lambda$ CDM model ${ }^{3}$ and cross-correlated each of them using the templates with the same procedure applied to the data. The amplitude of the predicted chance correlation, given by the rms over the 1000 realizations, is $1.13 \mu \mathrm{K}_{\mathrm{CMB}} / \mu \mathrm{K}_{\mathrm{CMB}}$ for the dust template, $1.12 \mu \mathrm{K}_{\mathrm{CMB}} / \mathrm{R}$ for the free-free template and $3.8 \mu \mathrm{K}_{\mathrm{CMB}} / \mathrm{K}$ for the synchrotron template.

In the top panel of Fig. 8 we compare the $\mathrm{H} \alpha$ correlation coefficients (points with error bars) with the component separation results for free-free emission obtained in Sect. 5 (shaded

\footnotetext{
3 http://lambda.gsfc.nasa.gov/product/map/dr4/ pow_tt_spec_get.cfm
}
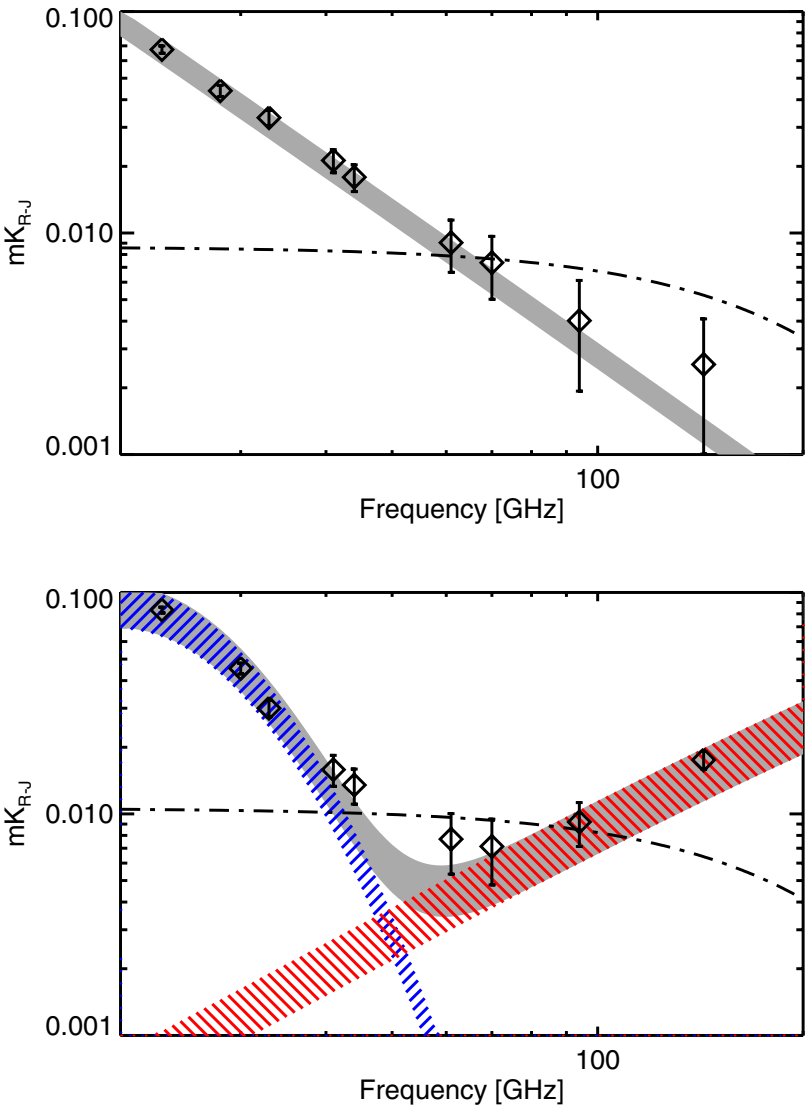

Fig. 8. Comparison between correlation coefficients (symbols with error bars) and component separation results (shaded areas) for free-free emission (top) and dust emission (bottom). For free-free emission we show the Dickinson et al. (2003) $\mathrm{H} \alpha$ correlation coefficients and for dust emission the Finkbeiner et al. (1999) correlation coefficients. The grey area in the bottom panel is the sum of the AME (blue) and thermal dust (red) components. The dash-dotted line in both panels shows the $1 \sigma$ error due to the chance correlation of CMB with foreground templates, estimated using simulations.

area). The flux for both the component separation and crosscorrelation was computed as the standard deviation of the maps (the separated free-free map and the scaled $\mathrm{H} \alpha$ template, respectively) because this is not affected by possible offsets between the Planck data and the $\mathrm{H} \alpha$ template. The two results generally agree well; in the frequency range $40-60 \mathrm{GHz}$ there is an excess in the correlated coefficients, which could be indicative of a contribution from the AME component (similar to that found by Dobler \& Finkbeiner 2008b). Flattening of the C-C coefficients for $v>60 \mathrm{GHz}$ is consistent with positive chance correlation between the $\mathrm{CMB}$ and the $\mathrm{H} \alpha$ template.

The dust-correlated coefficients are compared with the component separation results in the bottom panel of Fig. 8. The agreement is very good for $v<40 \mathrm{GHz}$ and $v>100 \mathrm{GHz}$, where AME and thermal dust emission are strong. In the $40-70 \mathrm{GHz}$ 
range the $\mathrm{C}-\mathrm{C}$ results are higher than the component separation results. As discussed in Appendix B, the parametric fit to the AME spectrum implemented by CCA could be inaccurate in this frequency range, where the AME is faint. Alternatively, a similar effect could be explained by the presence of a secondary AME peak, around $40 \mathrm{GHz}$ (e.g. Planck Collaboration 2011d; Ghosh et al. 2012) or flattening of the dust spectral index towards low frequencies, which are not included in our spectral model. Distinguishing between these hypotheses is not possible given the large error bars.

To determine the free-free electron temperature, the $\mathrm{H} \alpha$ correlation coefficients were fitted with a combination of powerlaw free-free radiation (with a fixed spectral index of -2.14) and a CMB chance correlation term (which is constant in thermodynamic units). The amplitude of the free-free component with respect to $\mathrm{H} \alpha$ resulting from the fit, and its uncertainty, yield $T_{\mathrm{e}}$ and the corresponding error bar. The results for the Gould Belt region outside the adopted sky mask are reported in the bottom part of Table 3 . We found $T_{\mathrm{e}}=5300-2400 \mathrm{~K}$ for $f_{\mathrm{d}}=0-0.5$ with the Dickinson et al. (2003) template, and $T_{\mathrm{e}}=7000-3800 \mathrm{~K}$ for $f_{\mathrm{d}}=0-0.5$ with the Finkbeiner (2003) template.

With respect to the T-T analysis, these results are more sensitive to the choice of the template and the $f_{\mathrm{d}}$ correction. Similarly, we expect the $\mathrm{C}-\mathrm{C}$ analysis to be more sensitive to the other systematic uncertainties on the templates, such as the contribution of scattered light to the $\mathrm{H} \alpha$ map (Witt et al. 2010; Brandt \& Draine 2012).

The sensitivity of the $\mathrm{C}-\mathrm{C}$ analysis to differences between the Dickinson et al. and Finkbeiner templates - the former yielding lower $T_{\mathrm{e}}$ than the latter - is a known problem (see Ghosh et al. 2012 for a detailed analysis). The different processing of the two maps results in residuals at the $1 \mathrm{R}$ level over large regions of the sky, and of more than $20 \mathrm{R}$ near very bright regions. The adopted $\chi^{2}$ estimator, which contains the square of the template in the denominator, tends to amplify the differences.

For this analysis we adopted a $3^{\circ}$ resolution, as advised by Ghosh et al. (2012) to reduce artefacts in the templates due to beam effects, and we masked the most discrepant pixels. Still, the best-fit electron temperatures yielded by the two templates may differ by $30 \%$, whereas for the T-T analysis this difference is $10 \%$ at most. In fact, the fit of the T-T plot is determined by large samples of pixels, on which the two templates are generally more similar, while the $\mathrm{C}-\mathrm{C}$ method is more sensitive to bright features, on which they may be more different. We verified that by enlarging the mask to exclude the brightest pixels, the numbers we obtain for the two templates agree better.

The $\mathrm{C}-\mathrm{C}$ results are always consistent with the $\mathrm{T}-\mathrm{T}$ ones within the error bars, but they are systematically lower for the Dickinson et al. template. In addition to systematic errors related to methods and templates, a difference between T-T and C-C results could also indicate spatial variability of $T_{\mathrm{e}}$ within the region, since the two methods have different sensitivity to different features in the map. This confirms that estimating the free-free electron temperature is a difficult problem and that caution is needed when interpreting the results.

\section{AME as spinning-dust emission}

An explanation that is often invoked for the AME is electric dipole radiation from small, rapidly spinning, polycyclic aromatic hydrocarbon (PAHs) dust grains (Erickson 1957; Draine \& Lazarian 1998; Dobler \& Finkbeiner 2008b; Dobler et al. 2009; Hoang et al. 2011).
Alternatively, the AME could be generated by synchrotron radiation with a flat (hard) spectral index (e.g. Bennett et al. 2003). The presence of such a hard spectrum synchrotron component could be highlighted by comparing the $408 \mathrm{MHz}$ map of Haslam et al. (1982), which would predominantly trace steep spectrum radiation, with the $2.3 \mathrm{GHz}$ map by Jonas et al. (1998), which would be more sensitive to flat spectrum radiation. This question has been studied in detail by Peel et al. (2012) using a cross-correlation of WMAP 7-yr data with foreground templates. They analysed the region defined by $170^{\circ} \leq l \leq 210^{\circ}$, $-55^{\circ} \leq b \leq-25^{\circ}$ and found that the dust-correlated coefficients are mostly unaffected by the use of the $2.3 \mathrm{GHz}$ template instead of the $408 \mathrm{MHz}$ template. This indicates that hard synchrotron radiation cannot account for most of the dust-correlated component at low frequencies.

To check the hypothesis of spinning dust emission we applied the method proposed by Ysard et al. (2011), which exploits the SpDust (Ali-Haïmoud et al. 2009; Silsbee et al. 2011) and DustEM (Compiègne et al. 2011) codes, to model the frequency spectra of thermal and anomalous dust emission from the microwaves to the IR. The dust populations and properties are assumed to be the same as in the diffuse interstellar medium at high Galactic latitude (DHGL), defined in Compiègne et al. (2010). This model includes three dust populations: PAHs; amorphous carbonaceous grains; and amorphous silicates. For PAHs, it assumes a log-normal size distribution with centroid $a_{0}=0.64 \mathrm{~nm}$ and width $\sigma=0.4$, with a dust-to-gas mass ratio $M_{\mathrm{PAH}} / M_{\mathrm{H}}=$ $7.8 \times 10^{-4}$.

By fitting the thermal dust spectrum with DustEM we determined the local intensity of the interstellar radiation field, $G_{0}$ (the scaling factor with respect to a UV flux of $1.6 \times$ $10^{-3} \mathrm{erg} \mathrm{s}^{-1} \mathrm{~cm}^{-2}$ integrated between 6 and $13.6 \mathrm{eV}$ ), and the hydrogen column density, $N_{\mathrm{H}}$. We then fitted the AME spectrum with SpDust, the only free parameter being the local hydrogen density $n_{\mathrm{H}}$. We assumed a cosmic-ray ionization rate $\zeta_{\mathrm{CR}}=5 \times 10^{-17} \mathrm{~s}^{-1} \mathrm{H}^{-1}$, and took the electric dipole moment to be the same as in Draine \& Lazarian (1998), a prescription also shown to be compatible with the AME extracted from WMAP data (Ysard et al. 2010). It is worth noticing that there is a degeneracy with the size of the grains (smaller size yields higher peak frequency and intensity of the AME). However, the size distribution can only be constrained using shorter-wavelength data (typically $3-8 \mu \mathrm{m})$. The size we adopted $(0.64 \mathrm{~nm})$ was motivated by its ability to reproduce the data in the mid-IR (Compiègne et al. 2011); other models adopted different sizes (e.g. $0.54 \mathrm{~nm}$ and $0.5 \mathrm{~nm}$ in Draine \& Li 2001 and 2007 respectively).

Because the Gould Belt region contains strong foregroundemission components, which are significantly correlated with each-other, we expect different environments to be mixed in a complex way. To obtain meaningful results for the physical modelling we tried to isolate sub-regions where single environments dominate. To first order, we can use the free-free emission as a tracer of the ionized gas environment, $\mathrm{CO}$ emission as a tracer of molecular gas, and associate the rest of the emission with the diffuse ISM. In Fig. 9 we schematically map the different environments by setting a threshold on the free-free emission coming from component separation, the $\mathrm{CO}$ emission from Planck, and the total foreground emission at $30 \mathrm{GHz}$. We identified two relatively big sub-regions (shown as circles in Fig. 9) as selections that are dominated by ionized gas and diffuse ISM environments. It would not be meaningful to consider smaller areas because of the patch-by-patch estimation of the AME frequency scalings, which means that our AME spectra are averaged over relatively large areas of the sky. Due to the clumpiness of the molecular gas 


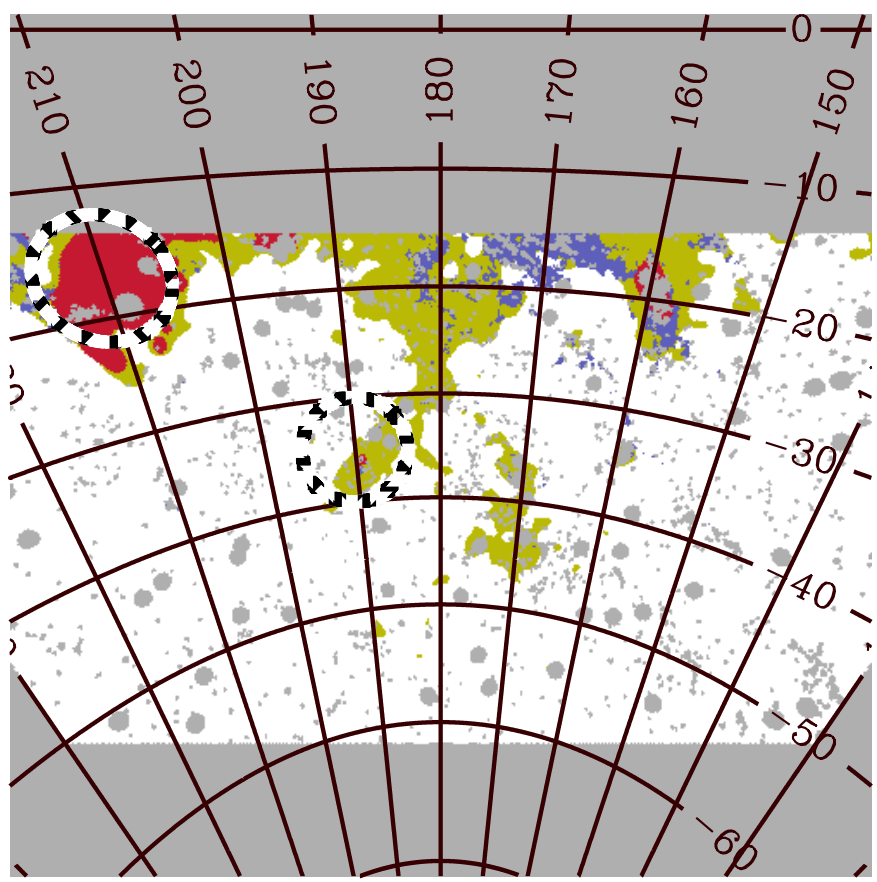

Fig. 9. Partitioning of the Gould Belt region based on thresholds over free-free emission (red), $\mathrm{CO}$ emission (blue), and total emission at $30 \mathrm{GHz}$ (yellow), used as tracers of $\mathrm{H} \mathrm{II} \mathrm{gas,} \mathrm{molecular} \mathrm{gas,} \mathrm{and} \mathrm{diffuse}$ ISM environments, respectively, and the rest of the Gould Belt region (white). Circled regions are those selected for computing the spectra and have been labelled H II-gas (Barnard's arc) and diffuse ISM (centred on $l=190^{\circ}, b=-35^{\circ}$ ) regions, respectively.

environment it was not possible to select a region for this case. It is worth noting that some molecular gas may be contained in the diffuse ISM region.

The spectra of the AME and thermal dust in the $20-353 \mathrm{GHz}$ frequency range are based on component-separation results. The frequency scaling is that estimated with CCA and the normalization is given by the average of the reconstructed amplitude map in the region of the sky considered. The error bars on the data points include the rms of the amplitude in the same region (considered as the error in the normalization) and the errors on the estimated spectral parameters. The thermal dust spectra were complemented with higher-frequency data points computed directly from the frequency maps: Planck $545 \mathrm{GHz}$ and $857 \mathrm{GHz}$; IRIS $100 \mu \mathrm{m}$ map; and the IRIS $12 \mu \mathrm{m}$ map corrected for zodiacal light emission used in Ysard et al. (2010).

The results of the modelling for the ionized gas and diffuse ISM regions within the Gould Belt are shown in Fig. 10. The empirical spectra of AME coming from component separation can be successfully modelled as spinning-dust emission for both regions. The match between data and model becomes poorer at higher frequencies, where the AME spectrum could be biased (see Sect. C and Appendix B).

The joint fit of thermal and spinning-dust models yields plausible physical descriptions of the two environments. In the top panel of Fig. 10 the diffuse ISM region is modelled with $N_{\mathrm{H}}=$ $2.46 \times 10^{21} \mathrm{H} \mathrm{cm}^{-2}, G_{0}=0.55$ and $n_{\mathrm{H}}=50 \mathrm{~cm}^{-3}$. The ionized region (middle panel) is modelled with $N_{\mathrm{H}}=5.73 \times 10^{21} \mathrm{H} \mathrm{cm}^{-2}$, $G_{0}=0.90$ and $n_{\mathrm{H}}=25 \mathrm{~cm}^{-3}$.

We tested the stability of these results against calibration errors on the high-frequency Planck (545 and $857 \mathrm{GHz}$ ) and IRIS (100 $\mu \mathrm{m}$ and $12 \mu \mathrm{m}$ ) data (the remaining data points come from
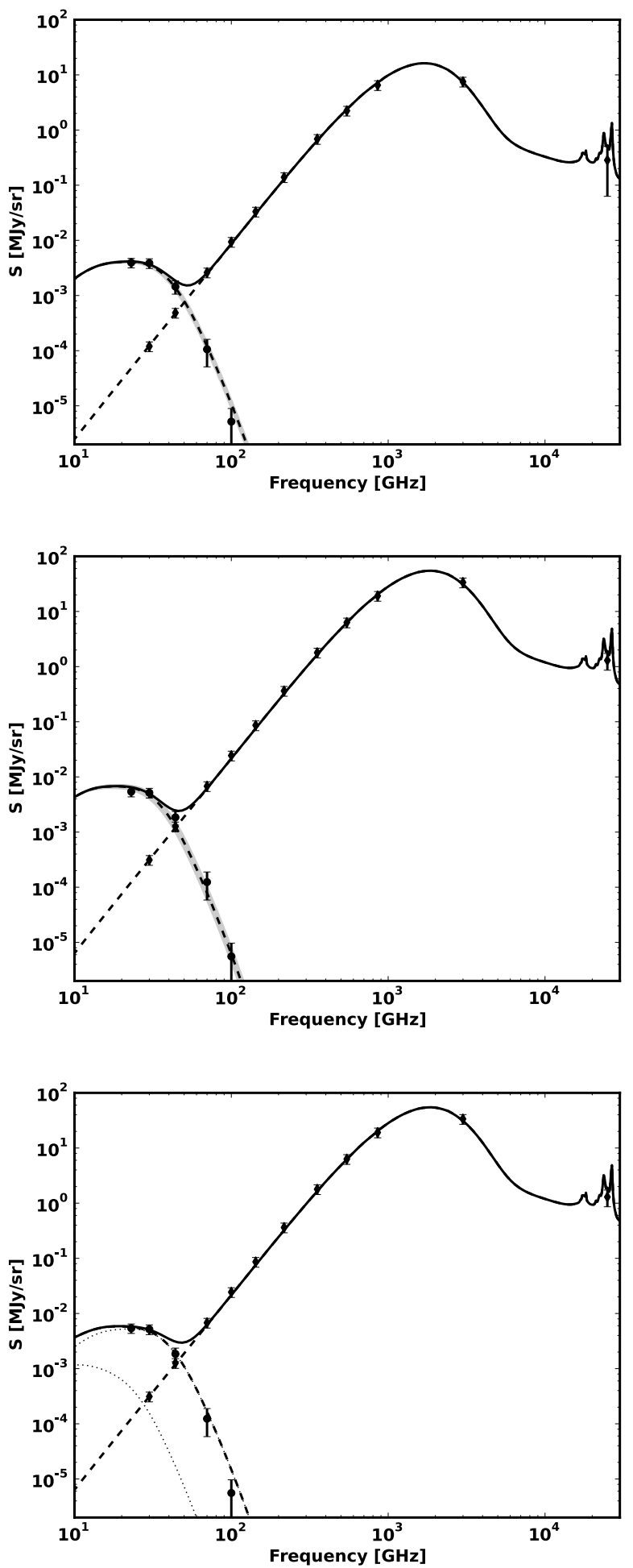

Fig. 10. Frequency spectra (black points with error bars) for thermal dust emission and AME compared, respectively, with DustEM and SpDust (dashed lines) for the diffuse ISM (top) and ionized gas (middle and bottom) regions within the Gould Belt. The solid line is the sum of the DustEM and SpDust models. The grey area in the top and middle panels corresponds to the $\pm 1 \sigma$ variations on the best-fit $n_{\mathrm{H}}$ values when fitting for a single phase. In the bottom panel we consider a mixture of two phases $\left(n_{\mathrm{H}}=0.1 \mathrm{~cm}^{-3}\right.$ and $n_{\mathrm{H}}=55 \mathrm{~cm}^{-3}$, in the proportion of $46 \%$ and $54 \%$, respectively), which marginally improves the fit for the ionized gas region at $23 \mathrm{GHz}$ (the error is $0.3 \sigma$ instead of $0.9 \sigma$ ).

the component-separation procedure and their error bars include systematic uncertainties). 
The total calibration uncertainty on the Planck 545 and $857 \mathrm{GHz}$ channels is estimated to be $10 \%$ (Planck Collaboration $2013 \mathrm{c}$ ), that on the IRIS data is of the order of $10 \%$ or larger, especially at $12 \mu \mathrm{m}$, where it also includes errors on the zodiacal light subtraction. We verified that very conservative uncertainties up to $20 \%$ both on Planck and IRIS data have a negligible impact on $G_{0}$, while they may affect $N_{\mathrm{H}}$ and $n_{\mathrm{H}}$ (up to a level of about $10 \%$ ). The overall picture does not change however: the ionized region is less dense and is illuminated by a stronger radiation field than the diffuse region (which is expected to contain mostly neutral gas). Both spectra can be modelled as spinningdust emission arising from regions with densities characteristic

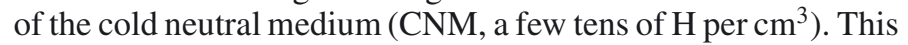
confirms the results of Planck Collaboration (2011d) and Planck Collaboration (2011e), showing that most of the observed AME could be explained by spinning dust in dense gas. In fact, whenever we have a mixture of warm neutral medium (WNM), warm ionized medium (WIM), and CNM, the spinning dust spectrum is dominated by the denser phase, which emits more strongly.

In the bottom panel of Fig. 10 we consider for the ionized region a mixture of two phases, one with a lower density $\left(n_{\mathrm{H}}=\right.$ $\left.0.1 \mathrm{~cm}^{-3}, 46 \%\right)$ and one with a higher density $\left(n_{\mathrm{H}}=55 \mathrm{~cm}^{-3}\right.$, $54 \%$ ), illuminated by the same $G_{0}$ as in the middle panel. This mixture fits the data somewhat better at $23 \mathrm{GHz}$ than the onephase model considered previously (the error in the fit at this frequency is $0.3 \sigma$ instead of $0.9 \sigma)$. To fully isolate and study different ISM phases (ionized/neutral, dense/diffuse), both the observations and the analysis should be carried out at high angular resolution.

\section{Conclusions}

We performed an analysis of the diffuse low-frequency Galactic foregrounds as seen by Planck in the southern part $\left(130^{\circ} \leq l \leq\right.$ $230^{\circ}$ and $-50^{\circ} \leq b \leq-10^{\circ}$ ) of the Gould Belt system, a local star-forming region emitting bright diffuse foreground emission. In addition to Planck data, our analysis included WMAP 7-yr data and foreground ancillary data as specified in Table 2.

We used the CCA (Bonaldi et al. 2006; Ricciardi et al. 2010) component separation method to separate the diffuse Galactic foregrounds. In the region of interest the synchrotron component is smooth and faint.

The free-free emission is strong and clearly dominates in the Orion-Barnard region. We inferred the free-free electron temperature both by cross-correlation (C-C) of channel maps with foreground templates and temperature-temperature (T-T) plots comparing the CCA free-free emission with $\mathrm{H} \alpha$ maps. We obtained $T_{\mathrm{e}}$ ranging from 3100 to $5200 \mathrm{~K}$ for $f_{\mathrm{d}}=0.3$, which broadens to $2400-7000 \mathrm{~K}$ when we allowed $f_{\mathrm{d}}$ to range within $0-0.5$. The Finkbeiner (2003) H $\alpha$ template yielded systematically higher $T_{\mathrm{e}}$ than the Dickinson et al. (2003) one. For the T-T analysis the difference is at most $500 \mathrm{~K}(<1 \sigma)$, while for the C-C analysis it can reach $2000 \mathrm{~K}$ (within $2 \sigma$ ). The $\mathrm{C}-\mathrm{C}$ results for the Dickinson et al. template are also systematically lower than the T-T ones, yet consistent within $1 \sigma$.

The AME is the dominant foreground emission at the lowest frequencies of Planck over most of the region considered. We estimated the AME peak frequency in flux density units to be $25.5 \pm 1.5 \mathrm{GHz}$, almost uniformly over the region of interest. This agrees with AME spectra measured in compact dust clouds (e.g. Planck Collaboration 2011d) and WMAP 9-yr results at low latitudes (once the same convention is adopted, e.g. their AME spectrum is converted from $\mathrm{K}_{\mathrm{R}-\mathrm{J}}$ to flux density, Bennett et al. 2012). For diffuse AME at higher latitudes a lower peak frequency is favoured (Banday et al. 2003; Davies et al. 2006; Ghosh et al. 2012; Bonaldi \& Ricciardi 2012). Spatial variability of the AME peak frequency is expected for spinning dust emission as a result of changes in the local physical conditions. For instance, the observed differences can be modelled in terms of a different density of the medium (lower density at high latitudes causes lower peak frequency) or a different size of the grains (smaller size giving higher peak frequency). The ability of our method to correctly recover the peak frequency of the AME, $v_{\mathrm{p}}$, was verified through realistic simulations. We also considered the effect of systematic errors in the spectral model and in the free-free template and demonstrated that they have only a negligible impact on $v_{\mathrm{p}}$.

Following Peel et al. (2012), a hard (flat spectrum) synchrotron component would not be sufficient to account for the dust-correlated low-frequency emission in this region. In support of the spinning dust mechanism, we performed a joint modelling of vibrational and rotational emission from dust grains as described by Ysard et al. (2011) and obtained a good description of the data from microwaves to the IR. The fit, which we performed separately for the ionized area near to Barnard's arc and the diffuse emission towards the centre of our region, in both cases yields plausible values for the local density and radiation field. This indicates that the spinning-dust mechanism can explain the AME in the Gould Belt reasonably.

Acknowledgements. Based on observations obtained with Planck (http:// www.esa.int/Planck), an ESA science mission with instruments and contributions directly funded by ESA Member States, NASA, and Canada. The development of Planck has been supported by: ESA; CNES and CNRS/INSUIN2P3-INP (France); ASI, CNR, and INAF (Italy); NASA and DoE (USA); STFC and UKSA (UK); CSIC, MICINN, JA and RES (Spain); Tekes, AoF and CSC (Finland); DLR and MPG (Germany); CSA (Canada); DTU Space (Denmark); SER/SSO (Switzerland); RCN (Norway); SFI (Ireland); FCT/MCTES (Portugal); and the development of Planck has been supported by: ESA; CNES and CNRS/INSU-IN2P3-INP (France); ASI, CNR, and INAF (Italy); NASA and DoE (USA); STFC and UKSA (UK); CSIC, MICINN and JA (Spain); Tekes, AoF and CSC (Finland); DLR and MPG (Germany); CSA (Canada); DTU Space (Denmark); SER/SSO (Switzerland); RCN (Norway); SFI (Ireland); FCT/MCTES (Portugal); and PRACE (EU). A description of the Planck Collaboration and a list of its members, including the technical or scientific activities in which they have been involved, can be found at http://www.sciops.esa.int/index.php ?project=planck\& page=Planck_Collaboration. We acknowledge the use of the HEALPix (Górski et al. 2005) package and of the LAMBDA website http://lambda. gsfc.nasa.gov.

\section{References}

Ali-Haïmoud, Y., Hirata, C. M., \& Dickinson, C. 2009, MNRAS, 395, 1055 Alves, M. I. R., Davies, R. D., Dickinson, C., et al. 2012, MNRAS, 422, 2429 AMI Consortium, Scaife, A. M. M., Hurley-Walker, N., et al. 2009, MNRAS, 400, 1394

Banday, A. J., Dickinson, C., Davies, R. D., Davis, R. J., \& Górski, K. M. 2003, MNRAS, 345, 897

Bedini, L., \& Salerno, E. 2007, Lecture Notes in Artificial Intelligence, 4964, 9 Bennett, C. L., Halpern, M., Hinshaw, G., et al. 2003, ApJS, 148, 1

Bennett, C. L., Larson, D., Weiland, J. L., et al. 2012, ApJS, submitted [arXiv: 1212.5225]

Bersanelli, M., Mandolesi, N., Butler, R. C., et al. 2010, A\&A, 520, A4

Bonaldi, A., \& Ricciardi, S. 2012, Adv. Astron., 2012, id. 853927

Bonaldi, A., Bedini, L., Salerno, E., Baccigalupi, C., \& de Zotti, G. 2006, MNRAS, 373, 271

Bonaldi, A., Ricciardi, S., Leach, S., et al. 2007, MNRAS, 382, 1791

Bond, J. R., \& Efstathiou, G. 1987, MNRAS, 226, 655

Boumis, P., Dickinson, C., Meaburn, J., et al. 2001, MNRAS, 320, 61

Brandt, T. D., \& Draine, B. T. 2012, ApJ, 744, 129

Broadbent, A., Osborne, J. L., \& Haslam, C. G. T. 1989, MNRAS, 237, 381

Casassus, S., Cabrera, G. F., Förster, F., et al. 2006, ApJ, 639, 951

Casassus, S., Dickinson, C., Cleary, K., et al. 2008, MNRAS, 391, 1075

Compiègne, M., Flagey, N., Noriega-Crespo, A., et al. 2010, ApJ, 724, L44 
Compiègne, M., Verstraete, L., Jones, A., et al. 2011, A\&A, 525, A103 Davies, R. D. 1960, MNRAS, 120, 483

Davies, R. D., Dickinson, C., Banday, A. J., et al. 2006, MNRAS, 370, 1125 de Oliveira-Costa, A., Tegmark, M., Davies, R. D., et al. 2004, ApJ, 606, L89 Dickinson, C. 2013, Adv. Astron., 2013, id. 162478

Dickinson, C., Davies, R. D., \& Davis, R. J. 2003, MNRAS, 341, 369 Dickinson, C., Casassus, S., Pineda, J. L., et al. 2006, ApJ, 643, L111 Dickinson, C., Davies, R. D., Bronfman, L., et al. 2007, MNRAS, 379, 297 Dickinson, C., Davies, R. D., Allison, J. R., et al. 2009, ApJ, 690, 1585

Dobler, G., \& Finkbeiner, D. P. 2008a, ApJ, 680, 1222

Dobler, G., \& Finkbeiner, D. P. 2008b, ApJ, 680, 1235

Dobler, G., Draine, B., \& Finkbeiner, D. P. 2009, ApJ, 699, 1374

Dong, R., \& Draine, B. T. 2011, ApJ, 727, 35

Draine, B. T., \& Lazarian, A. 1998, ApJ, 508, 157

Draine, B. T., \& Li, A. 2001, ApJ, 551, 807

Draine, B. T., \& Li, A. 2007, ApJ, 657, 810

Erickson, W. C. 1957, ApJ, 126, 480

Finkbeiner, D. P. 2003, ApJS, 146, 407

Finkbeiner, D. P., Davis, M., \& Schlegel, D. J. 1999, ApJ, 524, 867

Finkbeiner, D. P., Schlegel, D. J., Frank, C., \& Heiles, C. 2002, ApJ, 566, 898

Finkbeiner, D. P., Langston, G. I., \& Minter, A. H. 2004a, ApJ, 617, 350

Finkbeiner, D. P., Langston, G. I., \& Minter, A. H. 2004b, ApJ, 617, 350

Fixsen, D. J. 2009, ApJ, 707, 916

Ghosh, T., Banday, A. J., Jaffe, T., et al. 2012, MNRAS, 422, 3617

Giardino, G., Banday, A. J., Górski, K. M., et al. 2002, A\&A, 387, 82

Gold, B., Odegard, N., Weiland, J. L., et al. 2011, ApJS, 192, 15

Górski, K. M., Hivon, E., Banday, A. J., et al. 2005, ApJ, 622, 759

Gould, B. A. 1879, Resultados del Observatorio Nacional Argentino, 1, 1

Haslam, C. G. T., Salter, C. J., Stoffel, H., \& Wilson, W. E. 1982, A\&AS, 47, 1

Hoang, T., Lazarian, A., \& Draine, B. T. 2011, ApJ, 741, 87

Jarosik, N., Bennett, C. L., Dunkley, J., et al. 2011, ApJS, 192, 14

Jonas, J. L., Baart, E. E., \& Nicolson, G. D. 1998, MNRAS, 297, 977

Kogut, A. 1996, in BAAS, 28, 1295

Kogut, A., Fixsen, D. J., Levin, S. M., et al. 2011, ApJ, 734, 4

Lagache, G. 2003, A\&A, 405, 813

Lamarre, J., Puget, J., Ade, P. A. R., et al. 2010, A\&A, 520, A9

Leahy, J. P., Bersanelli, M., D’Arcangelo, O., et al. 2010, A\&A, 520, A8

Leitch, E. M., Readhead, A. C. S., Pearson, T. J., \& Myers, S. T. 1997, ApJ, 486, I 23

Lindblad, P. O. 1967, Bull. Astron. Inst. Netherlands, 19, 34

Lindblad, P. O., Palous, J., Loden, K., \& Lindegren, L. 1997, in Hipparcos Venice '97, eds. R. M. Bonnet, E. Høg, P. L. Bernacca, et al., ESA SP, 402, 507

Macellari, N., Pierpaoli, E., Dickinson, C., \& Vaillancourt, J. E. 2011, MNRAS, 418,888

Mandolesi, N., Bersanelli, M., Butler, R. C., et al. 2010, A\&A, 520, A3

Mennella, A., Bersanelli, M., Butler, R. C., et al. 2011, A\&A, 536, A3

Miville-Deschênes, M.-A., \& Lagache, G. 2006, in ASP Conf. Ser., 357, eds. L. Armus, \& W. T. Reach, 167

Miville-Deschênes, M.-A., Ysard, N., Lavabre, A., et al. 2008, A\&A, 490, 1093

Murphy, E. J., Helou, G., Condon, J. J., et al. 2010, ApJ, 709, L108

Peel, M. W., Dickinson, C., Davies, R. D., et al. 2012, MNRAS, 424, 2676

Perrot, C. A., \& Grenier, I. A. 2003, A\&A, 404, 519

Pietrobon, D., Górski, K. M., Bartlett, J., et al. 2012, ApJ, 755, 69

Planck Collaboration. 2011a, A\&A, 536, A1

Planck Collaboration. 2011b, A\&A, 536, A2

Planck Collaboration. 2011c, A\&A, 536, A19

Planck Collaboration. 2011d, A\&A, 536, A20

Planck Collaboration. 2011e, A\&A, 536, A21

Planck Collaboration. 2011f, A\&A, 536, A25

Planck Collaboration. 2013a, A\&A, submitted [arXiv: 1303.5063]

Planck Collaboration. 2013b, A\&A, submitted [arXiv: 1303.5067]

Planck Collaboration. 2013c, A\&A, submitted [arXiv: 1303.5070]

Planck Collaboration. 2013d, A\&A, 554, A139

Planck HFI Core Team. 2011a, A\&A, 536, A4

Planck HFI Core Team. 2011b, A\&A, 536, A6

Reynolds, R. J., \& Ogden, P. M. 1979, ApJ, 229, 942

Ricciardi, S., Bonaldi, A., Natoli, P., et al. 2010, MNRAS, 406, 1644

Rosset, C., Tristram, M., Ponthieu, N., et al. 2010, A\&A, 520, A13

Scaife, A., Green, D. A., Battye, R. A., et al. 2007, MNRAS, 377, L69

Scaife, A. M. M., Nikolic, B., Green, D. A., et al. 2010, MNRAS, 406, L45

Schlegel, D. J., Finkbeiner, D. P., \& Davis, M. 1998, ApJ, 500, 525

Silsbee, K., Ali-Haïmoud, Y., \& Hirata, C. M. 2011, MNRAS, 411, 2750

Tauber, J. A., Mandolesi, N., Puget, J., et al. 2010, A\&A, 520, A1

Tegmark, M., Eisenstein, D. J., Hu, W., \& de Oliveira-Costa, A. 2000, ApJ, 530, 133
Todorović, M., Davies, R. D., Dickinson, C., et al. 2010, MNRAS, 406, 1629 Watson, R. A., Rebolo, R., Rubiño-Martín, J. A., et al. 2005, ApJ, 624, L89

Witt, A. N., Gold, B., Barnes, III, F. S., et al. 2010, ApJ, 724, 1551

Wood, K., \& Reynolds, R. J. 1999, ApJ, 525, 799

Ysard, N., \& Verstraete, L. 2010, A\&A, 509, A12

Ysard, N., Miville-Deschênes, M. A., \& Verstraete, L. 2010, A\&A, 509, L1

Ysard, N., Juvela, M., \& Verstraete, L. 2011, A\&A, 535, A89

Zacchei, A., Maino, D., Baccigalupi, C., et al. 2011, A\&A, 536, A5

1 APC, AstroParticule et Cosmologie, Université Paris Diderot, CNRS/IN2P3, CEA/lrfu, Observatoire de Paris, Sorbonne Paris Cité, 10 rue Alice Domon et Léonie Duquet, 75205 Paris Cedex 13, France

2 Aalto University Metsähovi Radio Observatory, Metsähovintie 114, 02540 Kylmälä, Finland

3 African Institute for Mathematical Sciences, 6-8 Melrose Road, Muizenberg, Cape Town, South Africa

4 Agenzia Spaziale Italiana Science Data Center, c/o ESRIN, via Galileo Galilei, Frascati, Italy

5 Agenzia Spaziale Italiana, Viale Liegi 26, Roma, Italy

6 Astrophysics Group, Cavendish Laboratory, University of Cambridge, J J Thomson Avenue, Cambridge CB3 0HE, UK

7 CITA, University of Toronto, 60 St. George St., Toronto, ON M5S 3H8, Canada

8 CNR - ISTI, Area della Ricerca, via G. Moruzzi 1, Pisa, Italy

9 CNRS, IRAP, 9 Av. colonel Roche, BP 44346, 31028 Toulouse Cedex 4, France

10 California Institute of Technology, Pasadena, California, USA

11 Centro de Estudios de Física del Cosmos de Aragón (CEFCA), Plaza San Juan, 1, planta 2, 44001 Teruel, Spain

12 Computational Cosmology Center, Lawrence Berkeley National Laboratory, Berkeley, California, USA

13 Consejo Superior de Investigaciones Científicas (CSIC), Madrid, Spain

14 DSM/Irfu/SPP, CEA-Saclay, 91191 Gif-sur-Yvette Cedex, France

15 DTU Space, National Space Institute, Technical University of Denmark, Elektrovej 327, 2800 Kgs. Lyngby, Denmark

16 Département de Physique Théorique, Université de Genève, 24 Quai E. Ansermet, 1211 Genève 4, Switzerland

17 Departamento de Física Fundamental, Facultad de Ciencias, Universidad de Salamanca, 37008 Salamanca, Spain

18 Departamento de Física, Universidad de Oviedo, Avda. Calvo Sotelo s/n, Oviedo, Spain

19 Department of Astrophysics/IMAPP, Radboud University Nijmegen, PO Box 9010, 6500 GL Nijmegen, The Netherlands

20 Department of Electrical Engineering and Computer Sciences, University of California, Berkeley, California, USA

21 Department of Physics \& Astronomy, University of British Columbia, 6224 Agricultural Road, Vancouver, British Columbia, Canada

22 Department of Physics and Astronomy, Dana and David Dornsife College of Letter, Arts and Sciences, University of Southern California, Los Angeles, CA 90089, USA

23 Department of Physics, Gustaf Hällströmin katu 2a, University of Helsinki, Helsinki, Finland

24 Department of Physics, University of California, Santa Barbara, California, USA

25 Department of Physics, University of Illinois at Urbana-Champaign, 1110 West Green Street, Urbana, Illinois, USA

26 Dipartimento di Fisica e Astronomia G. Galilei, Università degli Studi di Padova, via Marzolo 8, 35131 Padova, Italy

27 Dipartimento di Fisica e Scienze della Terra, Università di Ferrara, via Saragat 1, 44122 Ferrara, Italy

28 Dipartimento di Fisica, Università La Sapienza, P. le A. Moro 2, Roma, Italy

29 Dipartimento di Fisica, Università degli Studi di Milano, via Celoria 16, Milano, Italy

30 Dipartimento di Fisica, Università degli Studi di Trieste, via A. Valerio 2, Trieste, Italy 
31 Dipartimento di Fisica, Università di Roma Tor Vergata, via della Ricerca Scientifica 1, Roma, Italy

32 Dipartimento di Matematica, Università di Roma Tor Vergata, via della Ricerca Scientifica 1, Roma, Italy

33 Discovery Center, Niels Bohr Institute, Blegdamsvej 17, Copenhagen, Denmark

34 Dpto. Astrofísica, Universidad de La Laguna (ULL), 38206 La Laguna, Tenerife, Spain

35 European Space Agency, ESAC, Planck Science Office, Camino bajo del Castillo, s/n, Urbanización Villafranca del Castillo, Villanueva de la Cañada, Madrid, Spain

36 European Space Agency, ESTEC, Keplerlaan 1, 2201 AZ Noordwijk, The Netherlands

37 Helsinki Institute of Physics, Gustaf Hällströmin katu 2, University of Helsinki, Helsinki, Finland

38 INAF - Osservatorio Astronomico di Padova, Vicolo dell'Osservatorio 5, Padova, Italy

39 INAF - Osservatorio Astronomico di Roma, via di Frascati 33, Monte Porzio Catone, Italy

40 INAF - Osservatorio Astronomico di Trieste, via G.B. Tiepolo 11, Trieste, Italy

41 INAF Istituto di Radioastronomia, via P. Gobetti 101, 40129 Bologna, Italy

42 INAF/IASF Bologna, via Gobetti 101, Bologna, Italy

43 INAF/IASF Milano, via E. Bassini 15, Milano, Italy

44 INFN, Sezione di Bologna, via Irnerio 46, 40126 Bologna, Italy

45 INFN, Sezione di Roma 1, Universit'a di Roma Sapienza, Piazzale Aldo Moro 2, 00185 Roma, Italy

46 IUCAA, Post Bag 4, Ganeshkhind, Pune University Campus, 411 007 Pune, India

47 Imperial College London, Astrophysics group, Blackett Laboratory, Prince Consort Road, London, SW7 2AZ, UK

48 Infrared Processing and Analysis Center, California Institute of Technology, Pasadena, CA 91125, USA

49 Institut Néel, CNRS, Université Joseph Fourier Grenoble I, 25 rue des Martyrs, Grenoble, France

50 Institut Universitaire de France, 103 bd Saint-Michel, 75005 Paris, France

51 Institut d'Astrophysique Spatiale, CNRS (UMR 8617) Université Paris-Sud 11, Bâtiment 121, Orsay, France

52 Institut d'Astrophysique de Paris, CNRS (UMR 7095), 98 bis Boulevard Arago, 75014 Paris, France

53 Institute for Space Sciences, Bucharest-Magurale, Romania

54 Institute of Astronomy and Astrophysics, Academia Sinica, Taipei, Taiwan

55 Institute of Astronomy, University of Cambridge, Madingley Road, Cambridge CB3 0HA, UK

56 Institute of Theoretical Astrophysics, University of Oslo, Blindern, Oslo, Norway

57 Instituto de Astrofísica de Canarias, C/Vía Láctea s/n, La Laguna, Tenerife, Spain

58 Instituto de Física de Cantabria (CSIC-Universidad de Cantabria), Avda. de los Castros s/n, Santander, Spain

59 Jet Propulsion Laboratory, California Institute of Technology, 4800 Oak Grove Drive, Pasadena, California, USA
60 Jodrell Bank Centre for Astrophysics, Alan Turing Building, School of Physics and Astronomy, The University of Manchester, Oxford Road, Manchester, M13 9PL, UK

61 Kavli Institute for Cosmology Cambridge, Madingley Road, Cambridge, CB3 OHA, UK

62 Kavli Institute for Theoretical Physics, University of California, Santa Barbara Kohn Hall, Santa Barbara, CA 93106, USA

63 LAL, Université Paris-Sud, CNRS/IN2P3, Orsay, France

${ }^{64}$ LERMA, CNRS, Observatoire de Paris, 61 Avenue de l'Observatoire, Paris, France

${ }^{65}$ Laboratoire AIM, IRFU/Service d'Astrophysique - CEA/DSM CNRS - Université Paris Diderot, Bât. 709, CEA-Saclay, 91191 Gif-sur-Yvette Cedex, France

${ }^{66}$ Laboratoire Traitement et Communication de l'Information, CNRS (UMR 5141) and Télécom ParisTech, 46 rue Barrault, 75634 Paris Cedex 13, France

${ }^{67}$ Laboratoire de Physique Subatomique et de Cosmologie, Université Joseph Fourier Grenoble I, CNRS/IN2P3, Institut National Polytechnique de Grenoble, 53 rue des Martyrs, 38026 Grenoble cedex, France

${ }^{68}$ Laboratoire de Physique Théorique, Université Paris-Sud 11 \& CNRS, Bâtiment 210, 91405 Orsay, France

69 Lawrence Berkeley National Laboratory, Berkeley, California, USA

70 Max-Planck-Institut für Astrophysik, Karl-Schwarzschild-Str. 1, 85741 Garching, Germany

71 MilliLab, VTT Technical Research Centre of Finland, Tietotie 3, Espoo, Finland

72 National University of Ireland, Department of Experimental Physics, Maynooth, Co. Kildare, Ireland

73 Niels Bohr Institute, Blegdamsvej 17, Copenhagen, Denmark

74 Optical Science Laboratory, University College London, Gower Street, London, UK

75 SISSA, Astrophysics Sector, via Bonomea 265, 34136 Trieste, Italy

76 School of Physics and Astronomy, Cardiff University, Queens Buildings, The Parade, Cardiff, CF24 3AA, UK

77 Space Sciences Laboratory, University of California, Berkeley, California, USA

78 Special Astrophysical Observatory, Russian Academy of Sciences, Nizhnij Arkhyz, Zelenchukskiy region, 369167 KarachaiCherkessian Republic, Russia

79 Stanford University, Dept of Physics, Varian Physics Bldg, 382 via Pueblo Mall, Stanford, California, USA

80 UPMC Univ Paris 06, UMR 7095, 98bis Boulevard Arago, 75014 Paris, France

81 Université de Toulouse, UPS-OMP, IRAP, 31028 Toulouse Cedex 4, France

82 Universities Space Research Association, Stratospheric Observatory for Infrared Astronomy, MS 232-11, Moffett Field, CA 94035, USA

83 University of Granada, Departamento de Física Teórica y del Cosmos, Facultad de Ciencias, Granada, Spain

84 University of Miami, Knight Physics Building, 1320 Campo Sano Dr. Coral Gables, Florida, USA

85 Warsaw University Observatory, Aleje Ujazdowskie 4, 00-478 Warszawa, Poland 


\section{Appendix A: Harmonic-domain CCA}

The sky radiation, $\tilde{x}$, from direction $r$ at frequency $v$ results from the superposition of signals coming from $N_{\mathrm{c}}$ different physical processes $\tilde{s}_{j}$ :

$$
\tilde{x}(r, v)=\sum_{j=1}^{N_{\mathrm{c}}} \tilde{s}_{j}(r, v) .
$$

The signal $\tilde{x}$ is observed through a telescope, the beam pattern of which can be modelled at each frequency as a spatially invariant point spread function $B(r, v)$. For each value of $v$, the telescope convolves the physical radiation map with $B$. The frequencydependent convolved signal is input to an $N_{\mathrm{d}}$-channel measuring instrument, which integrates the signal over frequency for each of its channels and adds noise to its outputs. The output of the measurement channel at a generic frequency $v$ is

$x_{v}(r)=\int B\left(r-r^{\prime}, v^{\prime}\right) \sum_{j=1}^{N_{\mathrm{c}}} t_{\nu}\left(v^{\prime}\right) \tilde{s}_{j}\left(r^{\prime}, v^{\prime}\right) \mathrm{d} r^{\prime} \mathrm{d} v^{\prime}+n_{v}(r)$,

where $t_{v}\left(v^{\prime}\right)$ is the frequency response of the channel and $n_{v}(r)$ is the noise map. The data model in Eq. (A.2) can be simplified by virtue of the following assumptions:

- Each source signal is a separable function of direction and frequency, i.e.,

$\tilde{s}_{j}(r, v)=s_{j}(r) f_{j}(v)$;

- $B(r, v)=B_{v}(r)$ is constant within the bandpass of the measurement channel.

These two assumptions lead us to a new data model:

$x_{v}(r)=B_{v}(r) * \sum_{j=1}^{N_{\mathrm{c}}} h_{v j} s_{j}(r)+n_{v}(r)$,

where $*$ denotes convolution, and

$h_{v j} \equiv \int t_{v}\left(v^{\prime}\right) f_{j}\left(v^{\prime}\right) \mathrm{d} v^{\prime}$.

For each location, $r$, we define

- the $N_{\mathrm{c}}$-vector $s$ (sources vector), whose elements are $s_{j}(r)$;

- the $N_{\mathrm{d}}$-vector $\boldsymbol{x}$ (data vector), whose elements are $x_{v}(r)$;

- the $N_{\mathrm{d}}$-vector $\boldsymbol{n}$ (noise vector), whose elements are $n_{v}(r)$;

- the diagonal $N_{\mathrm{d}}$-matrix $\mathbf{B}$, whose elements are $B_{v}(r)$;

- the $N_{\mathrm{d}} \times N_{\mathrm{c}}$ matrix $\mathbf{H}$, containing all $h_{v j}$ elements.

Then, we can rewrite Eq. (A.4) in vector form:

$\boldsymbol{x}(r)=[\mathbf{B} * \mathbf{H} \boldsymbol{s}](r)+\boldsymbol{n}(r)$.

The matrix $\mathbf{H}$ is called the mixing matrix and contains the frequency scaling of the components for all the data maps involved.

Under the assumption that $\mathbf{B}$ does not depend on the frequency, when working in the pixel domain, we can simplify Eq. (A.6) to

$\boldsymbol{x}=\mathbf{H} \boldsymbol{s}+\boldsymbol{n}$,

where the components in the source vector $s$ are now convolved with the instrumental beam.
Equation (A.6) can be translated into the harmonic domain, where for each transformed mode it becomes

$\boldsymbol{X}=\widetilde{\mathbf{B}} \mathbf{H} \boldsymbol{S}+\boldsymbol{N}$

where $\boldsymbol{X}, \boldsymbol{S}$, and $\boldsymbol{N}$ are the transforms of $\boldsymbol{x}, \boldsymbol{s}$, and $\boldsymbol{n}$, respectively, and $\widetilde{\mathbf{B}}$ is the transform of matrix $\mathbf{B}$. Relying on this data model, we can derive the following relation between the cross-spectra of the data $\widetilde{\mathbf{C}}_{\boldsymbol{x}}(\ell)$, sources $\widetilde{\mathbf{C}}_{\boldsymbol{s}}(\ell)$ and noise, $\widetilde{\mathbf{C}}_{\boldsymbol{n}}(\ell)$, all depending on the multipole $\ell$ :

$\widetilde{\mathbf{C}}_{x}(\ell)=\widetilde{\mathbf{B}}(\ell) \mathbf{H} \widetilde{\mathbf{C}}_{s}(\ell) \mathbf{H}^{\mathrm{T}} \widetilde{\mathbf{B}}^{\dagger}(\ell)+\widetilde{\mathbf{C}}_{n}(\ell)$

where the dagger superscript denotes the adjoint matrix. To reduce the number of unknowns, the mixing matrix is parametrized through a parameter vector $\boldsymbol{p}($ such that $\mathbf{H}=\mathbf{H}(\boldsymbol{p}))$, using the fact that its elements are proportional to the spectra of astrophysical sources (see Sect. 3.2).

Since the foreground properties are expected to be spatially variable, we work on relatively small square patches of data. This allows us to use the 2D Fourier transform to approximate the harmonic spectra (see, e.g., Bond \& Efstathiou 1987).

The HEALPix (Górski et al. 2005) data on the sphere are projected on the plane tangential to the centre of the patch and are re-gridded with a suitable number of bins to correctly sample the original resolution. Each pixel in the projected image is associated with a specific vector normal to the tangential plane and assumes the value of the HEALPix pixel nearest to the corresponding position on the sphere. Clearly, the projection and re-gridding process will create some distortion in the image at small scales and will modify the noise properties. However, we verified that this has only a negligible impact on the spectra in Eq. (A.9) for the scales considered in this work and, therefore, on the spectral parameters. If $\boldsymbol{x}(i, j)$ contains the data projected on the planar grid and $\boldsymbol{X}(i, j)$ is its two-dimensional discrete Fourier transform, the energy of the signal at a certain scale, which corresponds to the power spectrum, can be obtained as the average of $\boldsymbol{X}(i, j) \boldsymbol{X}^{\dagger}(i, j)$ over annular bins $D_{\hat{\ell}}, \hat{\ell}=1, \ldots, \hat{\ell}_{\max }$ (Bedini \& Salerno 2007):

$\widetilde{\mathbf{C}}_{\boldsymbol{x}}(\hat{\ell})=\frac{1}{M_{\hat{\ell}}} \sum_{i, j \in D_{\hat{\ell}}} \boldsymbol{X}(i, j) \boldsymbol{X}^{\dagger}(i, j)$,

where $M_{\hat{\ell}}$ is the number of pairs $(i, j)$ contained in the spectral bin denoted by $D_{\hat{\ell}}$. Every spectral bin $\hat{\ell}$ is related to a specific $\ell$ in the spherical harmonic domain by

$\ell=(\hat{\ell}-1) 2 p \Delta_{\ell} / N_{\text {pix }}$,

where $p$ is the thickness of the annular bin, $\Delta_{\ell}=180 / L_{\operatorname{deg}}\left(N_{\text {pix }}-\right.$ 1 ), and $L_{\text {deg }}, N_{\text {pix }}$ are the size in degrees and the number of pixels on the side of the square patch, respectively.

If we reorder the matrices $\mathbf{C}_{\boldsymbol{x}}(\hat{\ell})-\mathbf{C}_{\boldsymbol{n}}(\hat{\ell})$ and $\mathbf{C}_{\boldsymbol{s}}(\hat{\ell})$ into vectors $\boldsymbol{d}(\hat{\ell})$ and $\boldsymbol{c}(\hat{\ell})$, respectively, we can rewrite Eq. (A.9) as

$\boldsymbol{d}(\hat{\ell})=\mathbf{H}_{k}(\hat{\ell}) \boldsymbol{c}(\hat{\ell})+\boldsymbol{\epsilon}(\hat{\ell})$,

where $\mathbf{H}_{k}(\hat{\ell})=[\widetilde{\mathbf{B}}(\hat{\ell}) \mathbf{H}] \otimes[\widetilde{\mathbf{B}}(\hat{\ell}) \mathbf{H}]$, and the symbol $\otimes$ denotes the Kronecker product. The vector $\boldsymbol{d}(\hat{\ell})$ is now computed using the approximated data cross-spectrum matrix in Eq. (A.10) and $\boldsymbol{\epsilon}(\hat{\ell})$ represents the error on the noise power spectrum.

The parameter vector $\boldsymbol{p}$ and the source cross-spectra are finally obtained by minimizing the functional

$\boldsymbol{\Phi}\left[\boldsymbol{p}, \boldsymbol{c}_{V}\right]=$

$\left[\boldsymbol{d}_{V}-\mathbf{H}_{k B}(\boldsymbol{p}) \cdot \boldsymbol{c}_{V}\right]^{\mathrm{T}} \mathbf{N}_{\epsilon B}^{-1}\left[\boldsymbol{d}_{V}-\mathbf{H}_{k B}(\boldsymbol{p}) \cdot \boldsymbol{c}_{V}\right]+\lambda \boldsymbol{c}_{V}^{\mathrm{T}} \mathbf{C} \boldsymbol{c}_{V}$. 
A\&A 557, A53 (2013)

Table B.1. SpDust input parameters for the spectra in Fig. B.1 and best-fit parameters for the CCA spectral model.

\begin{tabular}{lccccccc}
\hline \hline & SpDust & & & & \multicolumn{3}{c}{ CCA } \\
Model name & $n_{\mathrm{H}}\left[\mathrm{cm}^{-3}\right]$ & $T[\mathrm{~K}]$ & $\chi$ & $x_{\mathrm{H}}$ & $x_{\mathrm{C}}$ & $v_{\mathrm{p}}$ & $m_{60}$ \\
\hline WNM & 0.4 & 6000 & 1.00 & 0.10 & 0.0003 & 24.22 & 7.53 \\
CNM & 30.0 & 100 & 1.00 & 0.0012 & 0.0003 & 29.00 & 4.93 \\
WIM & 0.1 & 8000 & 1.00 & 0.99 & 0.001 & 27.30 & 5.66 \\
MC & 300 & 20 & 0.01 & 0.0 & 0.0001 & 38.77 & 2.00 \\
\hline
\end{tabular}

Notes. The SpDust input parameters are the total hydrogen number density $n_{\mathrm{H}}$, the gas temperature $T$, the intensity of the radiation field relative to the average interstellar radiation field $\chi$, the hydrogen ionization fraction $x_{\mathrm{H}}=n_{\mathrm{H}^{+}} / n_{\mathrm{H}}$, and the ionized carbon fractional abundance $x_{\mathrm{C}}=n_{\mathrm{C}^{+}} / n_{\mathrm{H}}$.

The vectors $\boldsymbol{d}_{V}$ and $\boldsymbol{c}_{V}$ contain the elements $\boldsymbol{d}(\hat{\ell})$ and $\boldsymbol{c}(\hat{\ell})$, respectively, and the diagonal matrices $\mathbf{H}_{k B}$ and $\mathbf{N}_{\epsilon}$ the elements $\mathbf{H}_{k}(\hat{\ell})$ and the covariance of error $\boldsymbol{\epsilon}(\hat{\ell})$ for all relevant spectral bins. The term $\lambda \boldsymbol{c}_{V}^{\mathrm{T}} \mathbf{C} \boldsymbol{c}_{V}$ is a quadratic stabilizer for the source power cross-spectra: the matrix $\mathbf{C}$ is in our case the identity matrix, and the parameter $\lambda$ must be tuned to balance the effects of data fit and regularization in the final solution. The functional in Eq. (A.13) can be considered as a negative joint log-posterior for $\boldsymbol{p}$ and $\boldsymbol{c}_{V}$, where the first quadratic form represents the loglikelihood, and the regularization term can be viewed as a logprior density for the source power cross-spectra.

\section{Appendix B: Spectral model for AME}

Theoretical spinning-dust models predict a variety of spectra that can be substantially different in shape, depending on a large number of parameters describing the physics of the medium. The number of these physical parameters is too large to be constrained by the data in the available frequency range. For the purpose estimating the spectral behaviour of the AME we adopted a simple formula depending on only a few parameters. The CCA component-separation method used in this work implements the parametric relation proposed by Bonaldi et al. (2007) (Eq. (6)), depending on the peak frequency, $v_{\mathrm{p}}$, and slope at $60 \mathrm{GHz}, m_{60}$. To verify the adequacy of this parametrization we produced spinning-dust spectra for different input physical parameters with the SpDust code and fitted each of them with the proposed relation by minimizing the $\chi^{2}$ for the set of frequencies used in this work. The input models we considered are weak neutral medium (WNM), cold neutral medium (CNM), weak ionized medium (WIM), and molecular cloud (MC). Both the input SpDust parameters and the best-fit $m_{60}, v_{\mathrm{p}}$ parameters for each model are reported in Table B.1. For comparison, we also considered alternative parametric relations and in particular

- the model implemented in the Commander component separation method (Pietrobon et al. 2012; Planck Collaboration 2013d) which is a Gaussian in the $T_{\mathrm{CMB}}-\ln (v)$ plane, parametrized in terms of central frequency and width;

- the Tegmark et al. (2000) model, which is a modified blackbody relation (Eq. (1)) with a temperature of around $0.25 \mathrm{~K}$ and an emissivity index of about 2.4;

Because this test neither accounts for the presence of the other components and includes any data simulation, it verifies the intrinsic ability of the parametric model to reproduce the actual spectra. Realistic estimation errors for the CCA model are derived through simulations in Appendix C.

Figure B. 1 compares the input spectra with the best-fit models for the different parametrizations. In general, the fits are accurate at least up to $v=50-60 \mathrm{GHz}$, while at higher frequencies the parametric relations may not be able to reproduce the input
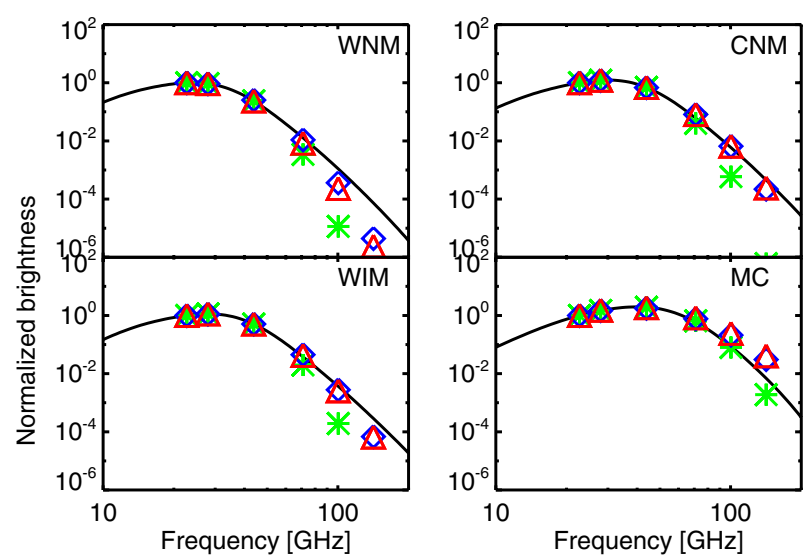

Fig. B.1. Theoretical spinning dust models produced with SpDust (solid lines) and fitted with CCA (triangles), Commander (diamonds), and Tegmark et al. (2000) (asterisks) models. Input SpDust parameters and best-fit parameters for the CCA model are provided in Table B.1.

spectra in detail. This is a consequence of fitting complex spectra with only a few parameters. The fit tends to fail where the AME signal is weaker.

Over the frequency range considered, CCA and Commander models fit the input spectrum generally better than the Tegmark et al. (2000) model (which decreases too rapidly at high frequencies). When adding lower frequency data, however, CCA and Commander models will be increasingly inaccurate, because they are symmetric with respect to the peak of the emission. The models implemented by CCA and Commander perform quite similarly, despite the different formulation. As a result, these methods are able to give consistent answers, which ensures consistency between different analyses within Planck (e.g. Planck Collaboration 2013d).

The CCA model used in this work provides a reasonable fit to theoretical spinning-dust models for a variety of physical conditions. The best-fit parameters that we obtain, reported in Table B.1, vary significantly from one input model to another and have a straightforward interpretation in terms of the spectrum.

\section{Appendix C: Description of the simulations}

We simulated Planck and WMAP 7-yr data by assuming monochromatic bandpasses positioned at the central frequency of the bands, Gaussian beams at the nominal values indicated in Tables 1 and 2, and Gaussian noise generated according to realistic, spatially varying noise rms. Our model of the sky consists of the following components:

- CMB emission given by the best-fit power spectrum model from WMAP 7-yr analyses; 

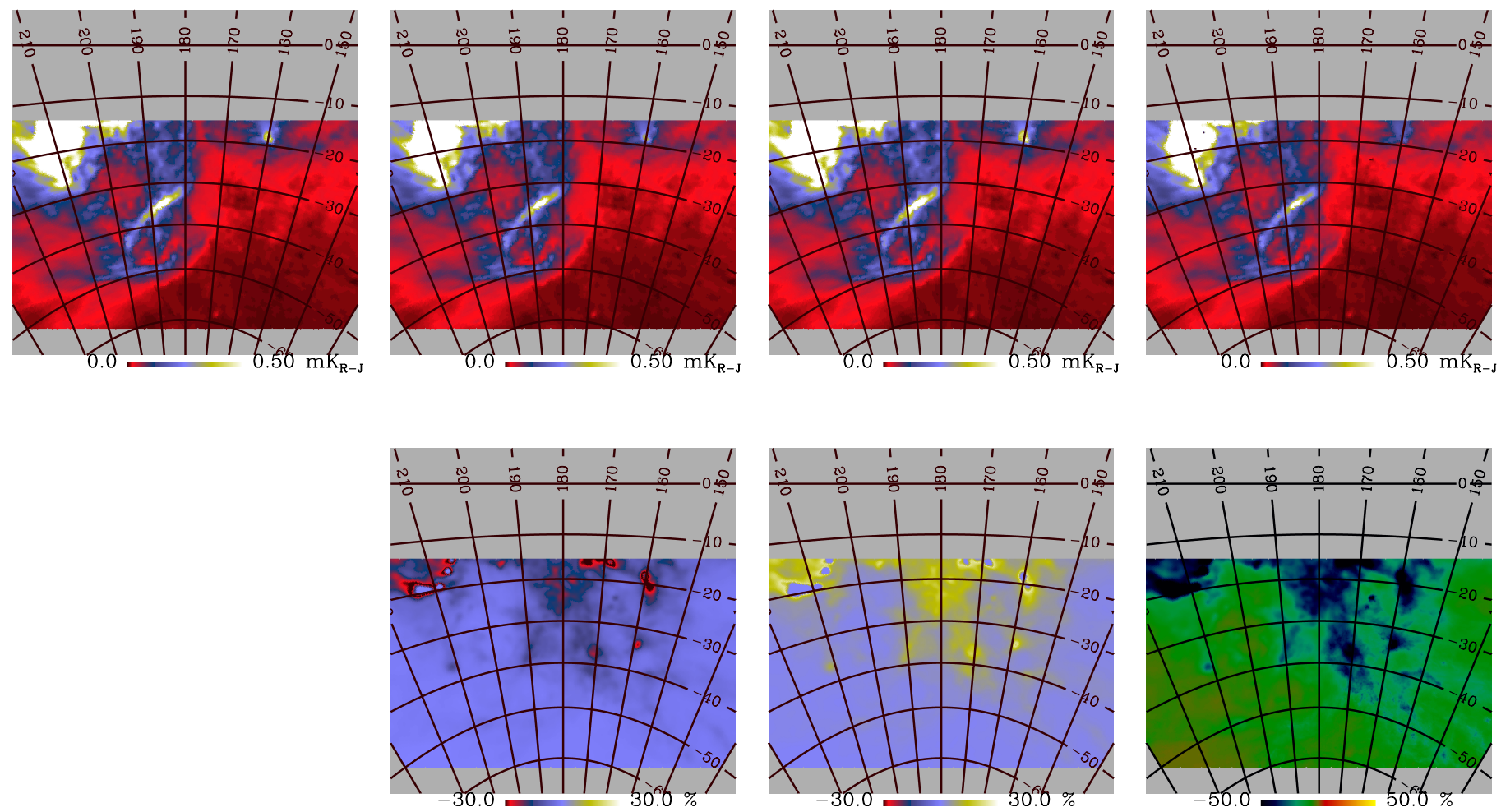

Fig. C.1. Free-free templates at $23 \mathrm{GHz}$ used for the analysis. The reference template $\mathrm{FF}_{\mathrm{REF}}$ is in the upper left corner; the other columns (left to right $)$ are $\mathrm{FF}_{1}, \mathrm{FF}_{2}$ and $\mathrm{FF}_{3}$, respectively. The differences in the lower panels $\left(\mathrm{FF}_{\mathrm{i}}-\mathrm{FF}_{\mathrm{REF}}\right) / \mathrm{FF}_{\mathrm{REF}}$ are on average of the order of $10 \%$, but reach $50 \%$ in regions of strong dust emission.

- synchrotron emission given by the Haslam et al. (1982) template scaled in frequency with a power-law model with a spatially varying synchrotron spectral index $\beta_{\mathrm{s}}$, as modelled by Giardino et al. (2002);

- free-free emission given by the Dickinson et al. (2003) $\mathrm{H} \alpha$ corrected for dust absorption with the $E(B-V)$ map from Schlegel et al. (1998) with a dust absorption fraction $f_{\mathrm{d}}=$ 0.33 , and scaled in frequency according to Eq. (2) with $T_{\mathrm{e}}=$ $7000 \mathrm{~K}$;

- thermal dust emission modelled with the $100 \mu \mathrm{m}$ map from Schlegel et al. (1998), scaled in frequency according to Eq. (1) with $T_{\mathrm{d}}=18 \mathrm{~K}$ and a spatially varying $\beta_{\mathrm{d}}$ with an average value of 1.7 ;

- AME modelled by the $E(B-V)$ map from Schlegel et al. (1998) with the intensity at $23 \mathrm{GHz}$ calibrated using the results of Ghosh et al. (2012) for the same region of the sky.

We adopted more than one spectral model for the AME. We first considered two convex spectra, generated with the SpDust code: one peaking around $26 \mathrm{GHz}$ and the other peaking around $19 \mathrm{GHz}$. We also tested a spatially varying power-law model (with spectral index of $-3.6 \pm 0.6$, Ghosh et al. 2012), which could result from the superposition of multiple convex components along the line of sight.

It is worth noting that the simulated sky is more complex than the model assumed in the component separation. This has been done intentionally, to reflect a more realistic situation. Another realistic feature we included are errors in the synchrotron and free-free templates. The spatial variability of the synchrotron spectral index modifies the morphology of the component with respect to that traced by the $408 \mathrm{MHz}$ map from Haslam et al. (1982). The use of $\mathrm{H} \alpha$ as a tracer of free-free emission is affected by even larger uncertainties. Our uncertainties on the dust absorption fraction $f_{\mathrm{d}}$ (estimated to be $f_{\mathrm{d}}=0.33_{-0.15}^{+0.10}$ at intermediate latitudes by Dickinson et al. 2003) and on the scattering of $\mathrm{H} \alpha$ photons from dust grains can create dust-correlated biases in the template. This is illustrated in Fig. C.1, where we compare different versions of the free-free template. $\mathrm{FF}_{\mathrm{REF}}$ is our reference template, adopted for the analysis of real data and for simulating the component, which is corrected for $f_{\mathrm{d}}=0.33$ as described in Dickinson et al. (2003). Two more templates $\left(\mathrm{FF}_{1}\right.$ and $\mathrm{FF}_{2}$ ) were obtained by correcting $\mathrm{H} \alpha$ for $f_{\mathrm{d}}=0.33-0.15$ and $f_{\mathrm{d}}=0.33+0.1( \pm 1 \sigma$ according to Dickinson et al. 2003) . A final template $\left(\mathrm{FF}_{3}\right)$ was obtained by correcting $\mathrm{FF}_{\mathrm{REF}}$ for scattered light at the $15 \%$ level by subtracting from the freefree map the $1 \mu \mathrm{m}$ map of Schlegel et al. (1998) multiplied by a suitable constant factor (Witt et al. 2010). Difference maps $\left(\mathrm{FF}_{\mathrm{i}}-\mathrm{FF}_{\mathrm{REF}}\right) / \mathrm{FF}_{\mathrm{REF}}$, presented in the lower panels of Fig. C.1, are on average of order of $10 \%$, but can be much higher (up to $50-60 \%$ ) in regions of strong dust emission.

When analysing the simulated data, we used both $\mathrm{FF}_{1}$ and $\mathrm{FF}_{2}$ as free-free templates in place of $\mathrm{FF}_{\mathrm{REF}}$, which corresponds to the simulated component. For synchrotron emission the morphological mismatch between the simulated component and the template was achieved by scaling the component from $23 \mathrm{GHz}$ to $408 \mathrm{MHz}$ with a spatially varying spectral index. The comparison between component and template is presented in Fig. C.2; the differences are of the order of $10 \%$. The simulated data-sets described above were analysed with the CCA method using the same procedure as applied to the real data; the results of this assessment are presented in Sect. 4.1. As a separate test, we verified the impact of the CMB component on the results for $v_{\mathrm{p}}$ and $m_{60}$. We generated 100 sets of mock data with the same foreground emission and different realizations of CMB and instrumental noise, and 

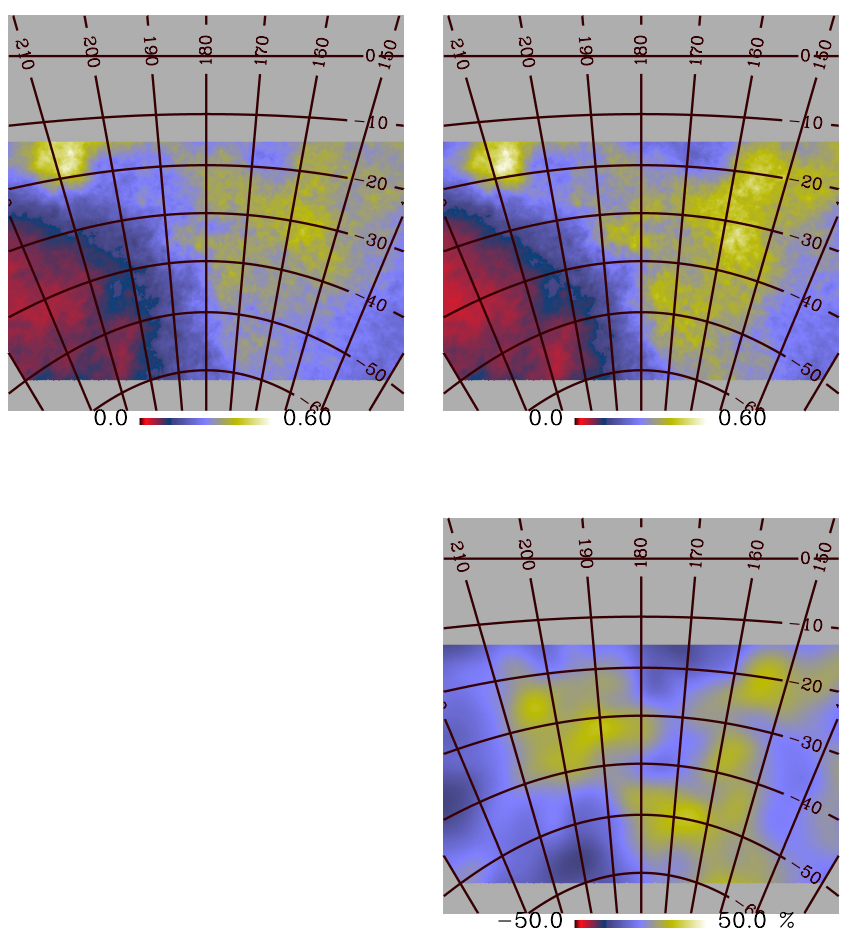

Fig. C.2. Upper panel: simulated synchrotron component (left) and synchrotron template (right) at $23 \mathrm{GHz}$. Lower panel: difference map divided by the simulated component.

repeated the estimation of the AME frequency scaling. For this test we used the simulation with a spatially constant AME spectrum peaking at $26 \mathrm{GHz}$. As this analysis is computationally demanding, the CCA estimation was performed only on the ten independent patches covering the Gould Belt region (centred on latitudes $-20^{\circ}$ and $-40^{\circ}$ and longitudes of $140^{\circ}, 160^{\circ}, 180^{\circ}$, $200^{\circ}$, and $220^{\circ}$ ). In Fig. C. 3 we show the average (diamonds) and rms (error bars) $v_{\mathrm{p}}$ and $m_{60}$ over the 100 realizations for each

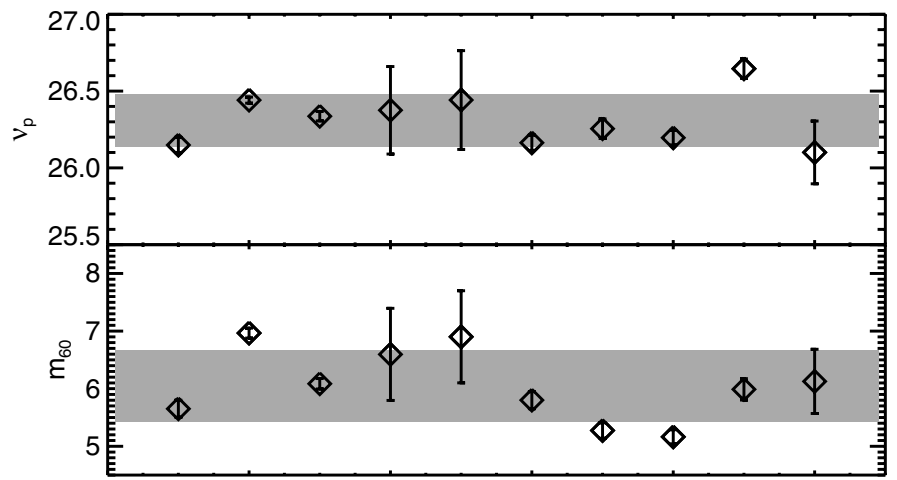

Sky patch

Fig. C.3. Average and rms of $v_{\mathrm{p}}$ and $m_{60}$ estimated over simulations with different $\mathrm{CMB}$ and noise realizations for different patches on the $x$-axis. The grey area is the average and rms over different patches, which is typically larger than that due to noise and CMB.

patch for different patches on the $x$-axis. The scatter between the results obtained for different patches (indicated by the grey area in the plots) is typically larger than the error bars, measuring the scatter due to different CMB realizations. This means that the foreground emission generally dominates over the CMB as a source of error. Larger error bars associated with the CMB are obtained for three patches with faint foreground emission. For these patches the estimated errors on $v_{\mathrm{p}}$ and $m_{60}$ are consistently larger. The CMB variation results on average in $\Delta v_{\mathrm{p}}=0.1 \mathrm{GHz}$ and $\Delta m_{60}=0.3$, which reach $0.3 \mathrm{GHz}$ and 0.8 , respectively, for the poorest sky patch. These values are below the error bars resulting from the analysis of the data, which amount to $1-1.5 \mathrm{GHz}$ for $v_{\mathrm{p}}$ and 1.5-2 for $m_{60}$. The CMB has limited impact on the results because this component is modelled in the mixing matrix. Having a known frequency scaling, the statistical constraint used by CCA is able to trace the pattern of the CMB through the frequencies with good precision and hence identify it correctly. 